\title{
Molecular Modeling and Synthesis of Ethyl Benzyl Carbamates as Possible Ixodicide Activity
}

\author{
Vázquez-Valadez Víctor Hugo', Hernández-S. Manuel Alejandro', \\ Velázquez-S. Ana María², Rosales-H. María ${ }^{3}$, Leyva-R. Marco Antonio3, \\ Prado-0. María Guadalupe ${ }^{4}$, Muñoz-G. Marco Antonio4, Alba-H. Fernando4, \\ Abrego Víctor5, Cruz-A. Diego², Ángeles Enrique ${ }^{1 *}$
}

\begin{abstract}
${ }^{1}$ Laboratorio de Química Medicinal, Departamento de Ciencias Químicas, FES-Cuautitlán UNAM, Edo. Mex., México ${ }^{2}$ Laboratorio de Investigación Nave 3000, Departamento de Ciencias Químicas, FES-Cuautitlán UNAM, Edo. Méx., México ${ }^{3}$ Departamento de Química, CINVESTAV, Cd. Mx., México

${ }^{4}$ Laboratorio 1 de la UIM, Departamento de Ciencias Biológicas, FES-Cuautitlán UNAM, Edo. Méx., México ${ }^{5}$ QSAR Analytics S.A. de C.V., Edo. Méx., México

Email: *angeles@unam.mx
\end{abstract}

How to cite this paper: Hugo, V.-V.V., Alejandro, H.-S.M., María, V.-S.A., María, R.-H., Antonio, L.-R.M., Guadalupe, P.-O.M., Antonio, M.-G.M., Fernando, A.-H., Víctor, A., Diego, C.-A. and Enrique, Á. (2019) Molecular Modeling and Synthesis of Ethyl Benzyl Carbamates as Possible Ixodicide Activity. Computational Chemistry, 7, 1-26. https://doi.org/10.4236/cc.2019.71001

Received: August 21, 2018

Accepted: December 18, 2018

Published: December 21, 2018

Copyright $\odot 2019$ by authors and Scientific Research Publishing Inc. This work is licensed under the Creative Commons Attribution International License (CC BY 4.0).

http://creativecommons.org/licenses/by/4.0/

\begin{abstract}
Carbamates are molecules that have different types of biological activities and provide a particular chemical control against ticks. The new structures of the proposed compounds were optimized and synthetized respectively, through a molecular model using the methods:PM3, HF and DFT applying the B3LYP functional, with the basis $6-31+\mathrm{G}(\mathrm{d})$ and $6-311+\mathrm{G}(\mathrm{d}, \mathrm{p})$, BVP86 and PBEPBE with $6-31+G(d)$ and the vibrational frequencies computed. These calculated frequencies were compared with the experimental ones to determine the most accurate level of theory for the prediction of vibrational frequencies of the compounds. The best results were obtained through HF/631+G(d). Additionally, we report a modification to obtain this type of compounds, and based on the amino-dehalogenation of ethyl chloroformate, different benzyl ethyl carbamates were synthesized modifying the base molecule. The performances obtained were compared to others already reported. The methodology used allowed us to synthesize new carbamates using benzylamine derivatives through a modification on the basic catalysis of the addition-elimination reaction.
\end{abstract}

\section{Keywords}

N-Carbamates, Ixodicide, Molecular Modeling

\section{Introduction}

The most important tick that parasitize cattle around the world is Rhipicephalus 
(Boophilus microplus), which is a hematophagous parasite that represents a great threat for cattle industry, not only because of the diseases it causes, but also for the huge economic loss it brings [1]. These losses are due to: Skin damage caused by parasite bites while feeding themselves (affecting negatively on the consumption value), anemia, transmitted pathologies, less food intake with consequential weight loss of cattle ( $0.6 \mathrm{~g}$ per each adult tick; between 40 and $60 \mathrm{Kg}$ of live weight per year), and therefore it diminishes milk and meat production; plus the extra costs for treatment on the affections caused by transmitted pathogens [2]. It is estimated that before its elimination in United States in 1943, the direct and indirect annual cost of babesiosis had a cost up to 130.5 million USD, equivalent to 3 thousand million dollars in 2007. Moreover, in Mexico, the loss is calculated to be approximately of up to 48 million USD per year.

Although there are different control measures, the parasite has not been eliminated in several countries, which are more prone to suffer from its consequences mainly due to the ecological conditions: Humidity, flora, temperature, innkeepers, etc. These helps for its survival, especially on tropical and subtropical zones; as well as the aimless use of anti-mites, which have been developed into the arrival of resistant strains to one or more kinds of ixodicides. An estimation of around 1,500,000 bovines is infected by the $R$. microplus and 500,000 more by the $R$. annulatus [3].

The detected resistance showed in Table 1 represents a high risk, due to the possibility of spread to the under control, the elimination and the free zones which would cause deep consequences for the chemical combat of ectoparasite and therefore for the impact of economy production and putting the cattle exports on hold for other countries [4] [5].

Table 1. Resistance to ixodicides by $R$. microplus.

\begin{tabular}{|c|c|c|}
\hline Substance & $\begin{array}{l}\text { Introduction } \\
\text { date }\end{array}$ & Location \\
\hline Arsenic & 1893 & $\begin{array}{l}\text { Australia, Argentina, 1936; Brazil, Colombia 1948; } \\
\text { Uruguay, 1953; Venezuela, } 1966 .\end{array}$ \\
\hline DDT & 1946 & $\begin{array}{l}\text { Argentina, Brazil, Australia, 1953; Venezuela, 1966; } \\
\text { South Africa, } 1979\end{array}$ \\
\hline $\begin{array}{l}\text { Toxaphene and } \\
\text { cyclodienes } \\
\text { (organochlorines) }\end{array}$ & 1947 & $\begin{array}{l}\text { Australia, Argentina, Brazil, 1953; Venezuela, } \\
\text { Colombia, 1966; South Africa, } 1979\end{array}$ \\
\hline $\begin{array}{l}\text { Organophosphates - } \\
\text { carbamates (anti-AChE) }\end{array}$ & 1953 & $\begin{array}{l}\text { Australia, Brazil, 1963; Argentina, 1964; Colombia, } \\
\text { Venezuela, 1967; South Africa, 1979; Uruguay, 1983; } \\
\text { México, } 1986\end{array}$ \\
\hline Formamidines & 1975 & $\begin{array}{l}\text { Australia, 1981; Brazil, 1995; Colombia, 2000; } \\
\text { México, } 2002\end{array}$ \\
\hline Pyrethroids & 1977 & $\begin{array}{l}\text { Australia, 1978; Brazil, 1989; México, 1994; } \\
\text { Venezuela, 1995; Colombia, 1997;Argentina, } 2000\end{array}$ \\
\hline Macrocyclic lactones & 1981 & Brazil, 2001; México, 2010 \\
\hline
\end{tabular}


Due to the great damages that the tick (Rhipicephalus microplus) brings to the cattle industry, millions of losses for wide distribution of the parasite and the resistance that mites have developed to all kinds of commercial ixodicide products on a national and international level [6]. The research and development of new anti-mites play an important role to fight and eliminate the ectoparasite. Today two derivative compounds from carbamic acid, have been identified as very effective for such purpose: Figure 1. Nevertheless, it is necessary to determine the stability of the compounds in a long term, as well as a more accurate pharmaceutical formula, its environmental impact, toxicity, among other factors [7]. Therefore, even there is a high possibility that the previous studies on carbamates could be useful for fighting the ectoparasite in a near future, it could not be used in case of being toxic or unsteady. Moreover, it is known that in average the resistance mechanism of the ticks is modified every 7 years. For those reasons, a new compound with effective results against ticks should be studied, nevertheless to synthetize new products and prove its utility seems expensive. On the other hand, such studies could be made in a theoretical way, using computational chemistry; this is a process which requires time but is addressed to the prediction of a great number of compounds that in case these turn out to be useful, they could have high probabilities of being approved for clinical use [8].

The carbamates which were developed and patented by our group, are 4-chlorophenylethyl carbamate (LQM996) and 4-bromophenylethyl carbamate (LQM919), which affected negatively to the biological parameters and reproduction of $R$. microplus by diminishing the oviposition, avoiding completely the viability of eggs and damaging the reproduction system of female. Likewise, in vitro experiments showed that carbamates interact very weakly with AChE and that its ixodicide activity is unrelated to such enzyme [7] [9]. The most outstanding fact is the efficiency in vivo shown by carbamates, being $99.9 \%$ for LQM 996 and $98.3 \%$ for LQM919. All of this was proved in a triple resistant strain to organophosphates pyrethroids and amidines.

The mechanism of the active carbamates like ixodicides is to disable the AChE enzyme. Nevertheless, the compounds synthetized by our group, do not act upon such enzyme. This result opens up the possibility to study the potential use of the derivatives from carbamic acid upon other targets. As a particular interest, the outstanding fact that the compounds LQM996 and LQM919 showed an unexpected result in vivo, causing the death of practically all the $R$. microplus larvae and affecting the reproduction system of the ticks as well as their eggs consistency. As this effect was not observed for the in vitro experiments, it could be caused by the action of carbamates upon a place that affects the reproductive system. Considering that sooner or later, parasite's nature will create a resistance, the intention of our group is to synthetize new possible alternatives by the time that the first resistant signs are detected, to rote the actives against the parasite. 


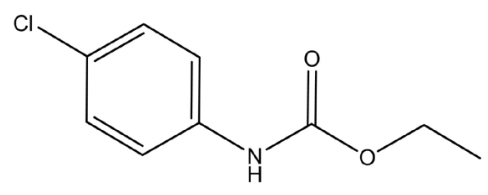

(a)

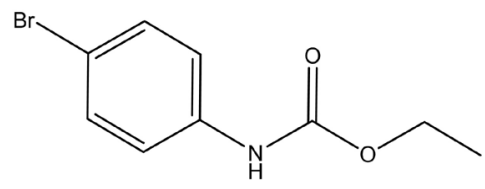

(b)

Figure 1. Carbamic acid derivatives with LQM key. (a) Ethyl (4-chlorophenyl) carbamate (LQM 996); (b) Ethyl (4-bromophenyl) carbamate (LQM 919).

The aim is to validate a theoretical method, this one should be capable to describe the theoretical and experimental frequencies (IR studies) of ethyl carbamates, and to compare the experimental angles obtained through X-ray diffraction with those found in the computational methods. Also, the descriptors such as Atomic Charges (ChelpG), Molecular Volume (Mv) and electronic descriptors correlated with $\mathrm{E}_{\text {номо }}$ and $\mathrm{E}_{\mathrm{LUMO}}$ values, such as molecular orbital energy gap $(\Delta \mathrm{E})$, chemical hardness $(\eta)$, chemical softness $(\sigma)$, chemical potential $(\mu)$, electrophilicity index $(\omega)$, electron affinity (A), ionization potential (I) and electronegativity $(\chi)$ were computed to corroborate which base is the more suitable for these compounds. The most suitable method will be used in the future for a QSAR study, to generate new molecules which could possess a high possibility to be useful and new efficient ixodicides.

\section{Experimental Procedures}

\subsection{Molecular Modeling of the New LQM9000 Series}

All calculations for the new compounds (described in Table 2) were carried out using Gaussian 09 [10] and GaussView 5.0. [11] The atomic and molecular properties were determined for the geometric optimization of the carbamates structures through the basis functions: $H F / 6-31+G(d)$, B3LYP/6-31+G(d), B3LYP/6-311+G(d,p), BVP86/6-31+G(d) and PBEPBE/6-31+G(d) [12] [13] [14] [15]. The molecules were initially minimize using the semi empiric method PM3, followed by the full optimization with the theory levels: $H F / 6-31+G(d)$, B3LYP/6-31+G(d), B3LYP/6-311+G(d,p), BVP86/6-31+G(d) and PBEPBE/6$31+G(d)$. The IR vibrations where computed as well. All basis methods add a diffuse function, allowing a better description of free electrons.

A comparison between experimental and theoretical data vibrations was done. Theoretical frequencies were corrected through an empiric scale factor specific for each method [16] due to the error caused by the harmonic proximity used during the calculation [12].

Following that, theoretical and experimental bond angles and bond distances of the LQM 9006 compound were compared using crystallographic data and geometric optimizations of the same basis mentioned before. Based on these comparisons, the absolute percentage error $\left(\% \mathrm{E}_{\mathrm{Abs}}\right)$ and average error was calculated for each compound and method. The method that better describes compounds geometry and frecuencies was determined based on the minor error percentage (Validation). 
Table 2. List of synthetized carbamates

ethylbenzylcarbamate

\subsection{Quantum Chemical Descriptors}

Calculation of molecular descriptors were made to the 5 new etyl carbamates compounds: Atomic Charges (ChelpG), Molecular Volume (Mv) chemical hardness $(\eta)$, chemical softness $(\sigma)$, chemical potencial $(\mu)$, electrophlicity index $(\omega)$, electron affinity $(\mathrm{A})$, ionization potential (I) and elecronegativity $(\chi)$. These parameteres were calculated using the appropiate relations. These studies were carried out with the same 5 different basis functions.

In order to compare theoretical methods, single point calculations were performed with atom geometry that comes from the crystallographic data obtained by X Ray diffraction of LQM 9006 compound, using the 5 basis functions mentioned above.

\subsection{LD50 Prediction Calculations}

For LD50 calculations, the QSAR Toolbox software was used. All molecular structures of all compounds were modeled using the Toolbox modeler tool, and all structures were double checked comparing each SMILES code. The profiling was carried out using: OECD HPV chemical categories, Substance type and 
US-EPA New Chemical Categories profiling methods. For category definition the Structure similarity over 50\% grouping method was applied.For LD50 prediction, the follow parameters were used: Human health hazard/Acute toxicity in Rat, 24 h duration, species: Rat; and Route of administration: oral. The descriptors used for the equation were: FM Reaction Time, Log Koa (Air-water partitioncoefficient model), Melting Point (Gold and Ogle Method) and Molecular Weight.

\subsection{Synthesis}

Benzylamine, ReagentPlus ${ }^{\varpi}$ 99\%. [100-46-9]. Aldrich Chemistry; Chlorobenzylamine, 95\%. [89-97-4] Sigma-Aldrich Co.; Chlorophormate of ethyl, 97\%. [541-41-3]. Sigma-Aldrich, Inc.; 4-Fluorobenzylamine, 97\%. [140-75-0]. Sigma-Aldrich Chemie GmbH.; 3,5-Bis(trifluoromethyl) benzylamine, $80 \%$. [85068-29-7] Sigma-Aldrich Co.; Carbonate of anhydrous potassium A.C.S. [584-08-7]. Golden Bell Reactives; Merck Kieselgel 60 F 254 precoated silica gel plates for TLC were obtained from $\mathrm{BDH}$.

The column chromatography was carried out with Merck 938S silica gel. Benzylamine or its derivative was dissolved in acetone and anhydrous $\mathrm{K}_{2} \mathrm{CO}_{3}$ were added and maintained with agitation between $-10^{\circ} \mathrm{C}$ to $0^{\circ} \mathrm{C}$. To this mixture, a solution of ethyl chlorophormate was added. The reaction was monitored through the TLC method, after ending the reaction, the solvent was eliminated, and it was recrystallized by $n$-hexane/acetone $95: 5$ which made a pure and corresponding product as a result.

\subsection{Characterization}

Infrared (IR) spectra was carried out in a ThermoFisher Scientific, Nicolet iS10; with Smart Orbit accessory, diamond optical window, in solid phase with a range from 3000 to $700 \mathrm{~cm}^{-1}$.

Proton NMR Spectrum $(300 \mathrm{MHz})$ and ${ }^{13} \mathrm{C}(75 \mathrm{MHz})$ were obtained with a Varian equipment, in $d$-DMSO and $\mathrm{CDCl}_{3}$, using TMS as an internal reference.

$\mathrm{X}$-Ray crystallography experiment of the compound LQM9006 was performed with an Enraf-Nonius CCD diffractometer, the radiation and wave length applied was MoK $\alpha$ con $\lambda=0.71073 \AA$ Á, hemisphere scanning type, $293.0 \mathrm{~K}$ for temperature, $2 \theta$ range for recollection of data from 8.24 to $54.90^{\circ}, 6439$ collected reflections $s^{*}, 3488$ (Rint $\left.=0.0200\right)$ independent reflections, the applied program for the solution of the structure is SHELXS-97 [18], refining method: Full-matrix Least-Squares on F. Mass spectrometry analysis was performed on JEOL AccuTOF JMS-T100LC. Ionization DART+and PEG-600 was used as internal standard.

\section{Results}

\subsection{Molecular Modeling}

The spectral data (vibrational frequencies) of the LQM compounds aresumma- 
rized in Tables 10-14: bond type that develops each vibrational band, experimental frequency $\left(v, \mathrm{~cm}^{-1}\right)$, theoretical frequencies and their respective scaled ( $v$ CORR, $\mathrm{cm}^{-1}$ ) as well the absolute percentage error (\%EAbs) for each theory level. According to the previous information: the average error, standard deviation, relative standard deviation percentage and "L" parameter which shows the spread of individual errors regarding the average (liability level 95\%), were calculated. The correction factors were used [16] [17] from the computational chemistry comparison and benchmark database.

Table 3 contains the average error calculated for each theory level, being $\mathrm{HF} / 6-31+\mathrm{G}(\mathrm{d})$ the basis which gave better results and a minor variation.

Comparison of bond angles and lengths: The LQM 9006 carbamate, showed in Figure 2, was the only compound that could be characterized through an $\mathrm{X}$ ray diffraction. The atomic resolution data obtained were used to compare the accuracy of theoretical optimized structures, the data are presented in Table 4 and Table 5.

The results for the quantum chemical descriptors are summarized in the Table 6.

\subsection{LD50 Prediction Calculations}

The LD50 prediction results are summarized in Table 7 and the statistical parameters are on Table 8 . The model equation found for prediction according to the parameters previously mentioned on section 2.2 is:

LD50 $=+1.71( \pm 0.19)+3.34 \mathrm{E}-04( \pm 3.6 \mathrm{E}-05) \times$ FM Reaction Time -0.0151 $( \pm 0.0177) \times \log$ Koa (Air-water partition coefficient model) $+1.20 \mathrm{E}-03$ $( \pm 1.49 \mathrm{E}-03) \times$ Melting Point (Gold and Ogle Method) $-1.11 \mathrm{E}-03( \pm 1.01 \mathrm{E}-03) \times$ Molecular weight, $\log (1 / \mathrm{mol} / \mathrm{kg})$

On Table 9 is showed the test set used to validate the QSAR prediction. 19 reported compounds were selected and calculated using the equation mentioned. After plotting the data from the Table 9 an $\mathrm{R}^{2}=0.9647$ was obtained [19]-[36].

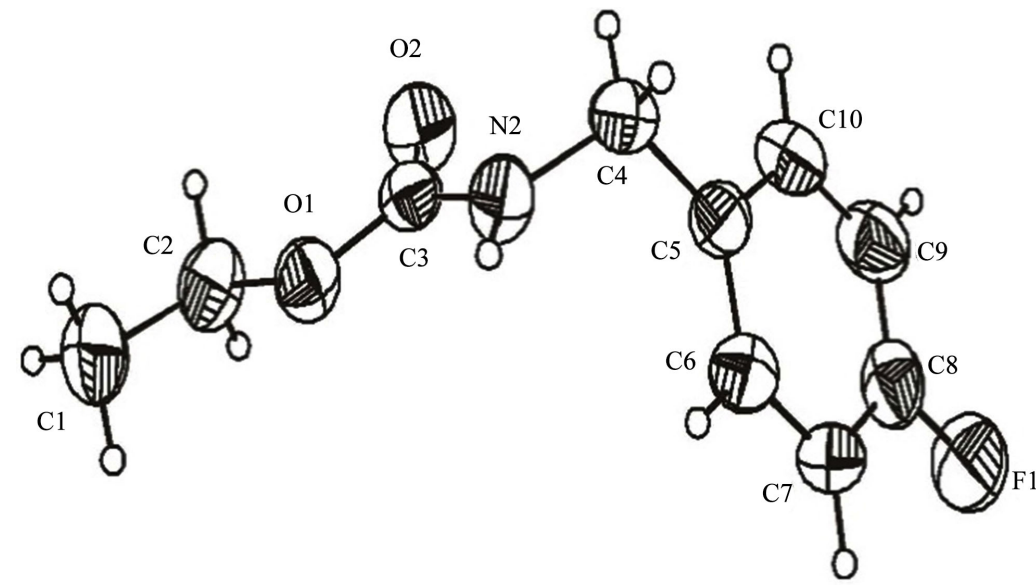

Figure 2. ORTEP representation of the LQM 9006 compound obtained through $\mathrm{X}$ ray diffraction. The labels shown are the same used in the result charts. 
Table 3. Average error and Standard Deviation of theory levels used.

\begin{tabular}{ccccccc}
\hline & PM3 & HF/6-31+G(d) & B3LYP/6-31+G(d,p) & B3LYP/6-311+G(d,p) & BVP86/6-31+G(d) & PBEPBE/6-31+G(d) \\
\hline Average & 4.53 & 1.88 & 2.28 & 2.72 & 3.32 & 2.57 \\
Std. Dev & 1.51 & 0.24 & 0.45 & 0.33 & 0.60 & 0.35 \\
\% RSD & 0.33 & 0.13 & 0.20 & 0.12 & 0.18 & 0.13 \\
L (0.05) & 1.32 & 0.21 & 0.40 & 0.29 & 0.52 & 0.30 \\
\hline
\end{tabular}

Table 4. Bond comparison of LQM 9006 (without H).

\begin{tabular}{|c|c|c|c|c|c|c|c|c|c|c|c|c|}
\hline Number & Bond & $\begin{array}{c}\text { Exp } \\
\text { distance } \\
\AA\end{array}$ & $\begin{array}{c}\text { Distance } \\
\mathrm{HF} / 6-31+\mathrm{G}(\mathrm{d})\end{array}$ & $\begin{array}{l}\text { \%Error } \\
\text { Abs. }\end{array}$ & $\begin{array}{c}\text { Distance } \\
\text { B3LYP/6-31+G(d) }\end{array}$ & $\begin{array}{c}\text { \%Error } \\
\text { Abs. }\end{array}$ & $\begin{array}{c}\text { Distance } \\
\text { B3LYP/6-311+G(d,p) }\end{array}$ & $\begin{array}{c}\text { \%Error } \\
\text { Abs. }\end{array}$ & $\begin{array}{c}\text { Distance } \\
\text { BVP86/6-31+G(d) }\end{array}$ & $\begin{array}{c}\text { \%Error } \\
\text { Abs. }\end{array}$ & $\begin{array}{c}\text { Distance } \\
\text { PBEPBE/6-31+G(d) }\end{array}$ & $\begin{array}{c}\text { \%Error } \\
\text { Abs. }\end{array}$ \\
\hline 1 & $\mathrm{C} 1 \mathrm{C} 2$ & 1.50 & 1.51 & 0.67 & 1.52 & 1.33 & 1.52 & 1.33 & 1.52 & 1.39 & 1.52 & 1.26 \\
\hline 2 & $\mathrm{C} 2 \mathrm{O} 1$ & 1.44 & 1.43 & 0.69 & 1.45 & 0.69 & 1.45 & 0.69 & 1.46 & 1.08 & 1.45 & 0.87 \\
\hline 3 & $\mathrm{C} 3 \mathrm{~N} 2$ & 1.32 & 1.35 & 2.27 & 1.36 & 3.03 & 1.36 & 3.03 & 1.37 & 4.03 & 1.37 & 3.97 \\
\hline 4 & $\mathrm{C} 3 \mathrm{O} 1$ & 1.34 & 1.33 & 0.75 & 1.36 & 1.49 & 1.36 & 1.49 & 1.37 & 2.48 & 1.37 & 2.37 \\
\hline 5 & $\mathrm{C} 3 \mathrm{O} 2$ & 1.23 & 1.20 & 2.44 & 1.22 & 0.81 & 1.21 & 1.63 & 1.23 & 0.19 & 1.23 & 0.15 \\
\hline 6 & C4 C5 & 1.52 & 1.52 & 0.00 & 1.52 & 0.00 & 1.52 & 0.00 & 1.52 & 0.12 & 1.52 & 0.02 \\
\hline 7 & $\mathrm{C} 4 \mathrm{~N} 2$ & 1.46 & 1.45 & 0.68 & 1.46 & 0.00 & 1.46 & 0.00 & 1.46 & 0.26 & 1.46 & 0.10 \\
\hline 9 & C6 C7 & 1.38 & 1.39 & 0.72 & 1.40 & 1.45 & 1.40 & 1.45 & 1.40 & 1.59 & 1.40 & 1.53 \\
\hline 10 & C7 C8 & 1.37 & 1.38 & 0.73 & 1.39 & 1.46 & 1.38 & 0.73 & 1.40 & 2.12 & 1.40 & 2.07 \\
\hline 11 & C8 C9 & 1.34 & 1.38 & 2.99 & 1.39 & 3.73 & 1.39 & 3.73 & 1.40 & 4.16 & 1.40 & 4.13 \\
\hline 12 & $\mathrm{C} 8 \mathrm{~F} 1$ & 1.36 & 1.33 & 2.21 & 1.36 & 0.00 & 1.36 & 0.00 & 1.37 & 0.64 & 1.37 & 0.51 \\
\hline 13 & C9 C10 & 1.38 & 1.38 & 0.00 & 1.40 & 1.45 & 1.39 & 0.72 & 1.41 & 1.85 & 1.40 & 1.78 \\
\hline Average & & & & 1.14 & & 1.30 & & 1.25 & & 1.71 & & 1.62 \\
\hline Std .Dev. & & & & 0.98 & & 1.11 & & 1.12 & & 1.33 & & 1.35 \\
\hline \% RSD & & & & 0.85 & & 0.85 & & 0.90 & & 0.78 & & 0.84 \\
\hline
\end{tabular}

Table 5. Angle comparison of LQM 9006.

\begin{tabular}{|c|c|c|c|c|c|c|c|c|c|c|c|c|}
\hline Number & Angle & $\begin{array}{c}\text { Exp. } \\
\text { Angle }(\AA ̊)\end{array}$ & $\begin{array}{l}\text { Angle HF/ } \\
6-31+G(d)\end{array}$ & $\begin{array}{c}\text { \%Error } \\
\text { Abs. }\end{array}$ & $\begin{array}{l}\text { Angle B3LYP/ } \\
6-31+G(d)\end{array}$ & $\begin{array}{c}\text { \%Error } \\
\text { Abs. }\end{array}$ & $\begin{array}{c}\text { Angle B3LYP/ } \\
6-311+G(d)\end{array}$ & $\begin{array}{c}\text { \%Error } \\
\text { Abs. }\end{array}$ & $\begin{array}{c}\text { Angle BVP86/ } \\
6-31+G(d)\end{array}$ & $\begin{array}{c}\text { \%Error } \\
\text { Abs. }\end{array}$ & $\begin{array}{l}\text { Angle PBEPBE/ } \\
6-31+\mathrm{G}(\mathrm{d})\end{array}$ & $\begin{array}{c}\text { \%Error } \\
\text { Abs. }\end{array}$ \\
\hline 1 & $\mathrm{H} 1 \mathrm{~A} \mathrm{C} 1 \mathrm{H} 1 \mathrm{C}$ & 109.50 & 108.36 & 1.04 & 108.29 & 1.10 & 108.28 & 1.12 & 108.22 & 1.16 & 108.22 & 1.17 \\
\hline 2 & $\mathrm{H} 1 \mathrm{~A} \mathrm{C} 1 \mathrm{C} 2$ & 109.40 & 109.70 & 0.27 & 109.58 & 0.16 & 109.60 & 0.19 & 109.53 & 0.12 & 109.56 & 0.15 \\
\hline 3 & $\mathrm{C} 1 \mathrm{C} 2 \mathrm{H} 2 \mathrm{~A}$ & 110.40 & 111.72 & 1.20 & 111.96 & 1.41 & 111.93 & 1.38 & 112.08 & 1.52 & 112.06 & 1.50 \\
\hline 4 & $\mathrm{C} 1 \mathrm{C} 2 \mathrm{O} 1$ & 106.60 & 107.35 & 0.70 & 107.43 & 0.78 & 107.46 & 0.81 & 107.32 & 0.68 & 107.36 & 0.71 \\
\hline 5 & $\mathrm{H} 2 \mathrm{~A} \mathrm{C} 2 \mathrm{H} 2 \mathrm{~B}$ & 108.60 & 108.23 & 0.34 & 107.98 & 0.57 & 107.87 & 0.67 & 107.88 & 0.66 & 107.79 & 0.74 \\
\hline 6 & $\mathrm{H} 2 \mathrm{~A} \mathrm{C} 2 \mathrm{O} 1$ & 110.40 & 108.89 & 1.37 & 108.74 & 1.50 & 108.82 & 1.43 & 108.73 & 1.51 & 108.79 & 1.46 \\
\hline 7 & $\mathrm{~N} 2 \mathrm{C} 3 \mathrm{O} 1$ & 111.80 & 110.94 & 0.77 & 110.21 & 1.42 & 110.09 & 1.53 & 109.72 & 1.86 & 109.74 & 1.84 \\
\hline
\end{tabular}




\section{Continued}

\begin{tabular}{|c|c|c|c|c|c|c|c|c|c|c|c|c|}
\hline 8 & $\mathrm{~N} 2 \mathrm{C} 3 \mathrm{O} 2$ & 124.00 & 125.08 & 0.87 & 125.42 & 1.15 & 125.56 & 1.25 & 125.62 & 1.31 & 125.58 & 1.27 \\
\hline 9 & $\mathrm{O} 1 \mathrm{C} 3 \mathrm{O} 2$ & 124.20 & 123.97 & 0.18 & 124.37 & 0.13 & 124.35 & 0.12 & 124.65 & 0.36 & 124.67 & 0.38 \\
\hline 10 & $\mathrm{H} 4 \mathrm{~A}$ C4 H4B & 107.80 & 107.51 & 0.27 & 107.67 & 0.12 & 107.62 & 0.17 & 107.72 & 0.07 & 107.69 & 0.11 \\
\hline 11 & $\mathrm{H} 4 \mathrm{~A}$ C4 C5 & 108.90 & 109.68 & 0.71 & 109.92 & 0.93 & 109.83 & 0.85 & 109.97 & 0.98 & 110.01 & 1.02 \\
\hline 12 & H4A C4 N2 & 108.80 & 107.80 & 0.92 & 108.05 & 0.69 & 108.27 & 0.49 & 108.54 & 0.24 & 108.42 & 0.35 \\
\hline 13 & C5 C4 N2 & 113.50 & 114.04 & 0.47 & 114.02 & 0.46 & 114.04 & 0.47 & 113.77 & 0.24 & 113.84 & 0.30 \\
\hline 14 & C4 C5 C6 & 121.10 & 120.92 & 0.15 & 120.85 & 0.20 & 120.87 & 0.19 & 120.91 & 0.15 & 120.94 & 0.13 \\
\hline 15 & C4 C5 C10 & 120.60 & 120.56 & 0.03 & 120.42 & 0.15 & 120.41 & 0.16 & 120.36 & 0.20 & 120.31 & 0.24 \\
\hline 16 & C6 C5 C10 & 118.00 & 118.52 & 0.44 & 118.72 & 0.61 & 118.72 & 0.61 & 118.73 & 0.62 & 118.74 & 0.63 \\
\hline 17 & C5 C6 H6 & 119.40 & 119.86 & 0.38 & 119.73 & 0.27 & 119.73 & 0.28 & 119.67 & 0.23 & 119.65 & 0.21 \\
\hline 18 & C5 C6 C7 & 121.10 & 121.33 & 0.19 & 121.24 & 0.11 & 121.21 & 0.09 & 121.25 & 0.13 & 121.25 & 0.12 \\
\hline 19 & C6 C7 C8 & 118.40 & 118.33 & 0.06 & 118.19 & 0.18 & 118.29 & 0.10 & 118.16 & 0.20 & 118.18 & 0.19 \\
\hline 20 & H7 C7 C8 & 120.70 & 120.00 & 0.58 & 120.00 & 0.58 & 119.92 & 0.64 & 120.03 & 0.55 & 120.00 & 0.58 \\
\hline 21 & C7 C8 C9 & 121.90 & 122.19 & 0.23 & 122.43 & 0.44 & 122.30 & 0.33 & 122.45 & 0.45 & 122.41 & 0.42 \\
\hline 22 & C7 C8 F1 & 118.40 & 119.01 & 0.51 & 118.82 & 0.35 & 118.90 & 0.42 & 118.82 & 0.35 & 118.83 & 0.36 \\
\hline 23 & C9 C8 F1 & 119.60 & 118.81 & 0.66 & 118.75 & 0.71 & 118.80 & 0.67 & 118.73 & 0.73 & 118.76 & 0.71 \\
\hline 24 & C8 C9 H9 & 120.20 & 119.78 & 0.35 & 119.84 & 0.30 & 119.75 & 0.37 & 119.88 & 0.27 & 119.84 & 0.30 \\
\hline 25 & C8 C9 C10 & 119.30 & 118.63 & 0.56 & 118.48 & 0.69 & 118.57 & 0.61 & 118.41 & 0.75 & 118.45 & 0.71 \\
\hline 26 & H9 C9 C10 & 120.40 & 121.59 & 0.99 & 121.69 & 1.07 & 121.67 & 1.06 & 121.71 & 1.09 & 121.71 & 1.08 \\
\hline 27 & C5 C10 C9 & 121.20 & 121.01 & 0.16 & 120.94 & 0.22 & 120.91 & 0.24 & 121.00 & 0.16 & 120.96 & 0.19 \\
\hline 28 & C5 C10 H10 & 119.30 & 119.54 & 0.20 & 119.43 & 0.11 & 119.44 & 0.11 & 119.41 & 0.09 & 119.37 & 0.06 \\
\hline 29 & C9 C10 H10 & 119.50 & 119.45 & 0.04 & 119.63 & 0.11 & 119.65 & 0.13 & 119.59 & 0.07 & 119.66 & 0.14 \\
\hline 30 & C3 N2 C4 & 123.20 & 122.64 & 0.46 & 122.12 & 0.87 & 122.12 & 0.87 & 121.83 & 1.11 & 121.69 & 1.23 \\
\hline 31 & C3 N2 H2 & 118.30 & 116.68 & 1.37 & 116.77 & 1.29 & 116.84 & 1.24 & 116.76 & 1.30 & 116.69 & 1.36 \\
\hline 32 & $\mathrm{C} 4 \mathrm{~N} 2 \mathrm{H} 2$ & 118.40 & 119.00 & 0.51 & 118.93 & 0.45 & 119.26 & 0.73 & 118.76 & 0.31 & 118.65 & 0.21 \\
\hline 33 & $\mathrm{C} 2 \mathrm{O} 1 \mathrm{C} 3$ & 116.90 & 117.46 & 0.48 & 115.91 & 0.85 & 115.98 & 0.79 & 114.91 & 1.70 & 114.86 & 1.74 \\
\hline Average & & & & 0.53 & & 0.61 & & 0.61 & & 0.64 & & 0.66 \\
\hline Std .Dev. & & & & 0.37 & & 0.44 & & 0.44 & & 0.53 & & 0.53 \\
\hline$\%$ RSD & & & & 0.70 & & 0.72 & & 0.71 & & 0.83 & & 0.81 \\
\hline L 0.05 & & & & 0.13 & & 0.15 & & 0.15 & & 0.18 & & 0.18 \\
\hline
\end{tabular}

Table 6. Descriptors calculated for the LQM studied.

\begin{tabular}{|c|c|c|c|c|c|c|c|c|c|c|c|c|}
\hline & & & $\mathrm{E}_{\text {номо }}(\mathrm{eV})$ & $\mathrm{E}_{\text {LUMO }}(\mathrm{eV})$ & $\Delta \mathrm{E}(\mathrm{eV})$ & $\mathrm{n}$ & $\sigma$ & $\omega$ & I & A & $\mu$ & $\mathrm{X}$ \\
\hline \multirow{8}{*}{$\begin{array}{l}\text { LQM } \\
9011\end{array}$} & \multirow{4}{*}{$\begin{array}{c}\text { Gas } \\
\text { Phase }\end{array}$} & pbepbe/6-31+G(d) & -0.1970 & -0.0402 & 0.1568 & 0.0784 & -12.7559 & 0.0897 & 0.1970 & 0.0402 & -0.1186 & 0.1186 \\
\hline & & $\mathrm{HF} / 6-31+\mathrm{G}(\mathrm{d})$ & -0.3097 & 0.0694 & 0.3791 & 0.1895 & -5.2759 & 0.0381 & 0.3097 & -0.0694 & -0.1202 & 0.1202 \\
\hline & & bvp86/6-31+G(d) & -0.1998 & -0.0428 & 0.1570 & 0.0785 & -12.7380 & 0.0937 & 0.1998 & 0.0428 & -0.1213 & 0.1213 \\
\hline & & B3LYP/6-31+G(d) & -0.2270 & -0.0129 & 0.2141 & 0.1070 & -9.3423 & 0.0672 & 0.2270 & 0.0129 & -0.1199 & 0.1199 \\
\hline & \multirow{4}{*}{ PCM } & B3LYP/6-311+G(d,p) & -0.2291 & -0.0155 & 0.2136 & 0.1068 & -9.3650 & 0.0700 & 0.2291 & 0.0155 & -0.1223 & 0.1223 \\
\hline & & pbepbe/6-31+G(d) & -0.2003 & -0.0437 & 0.1565 & 0.0783 & -12.7763 & 0.0951 & 0.2003 & 0.0437 & -0.1220 & 0.1220 \\
\hline & & $\mathrm{HF} / 6-31+\mathrm{G}(\mathrm{d})$ & -0.3151 & 0.0729 & 0.3880 & 0.1940 & -5.1553 & 0.0378 & 0.3151 & -0.0729 & -0.1211 & 0.1211 \\
\hline & & bvp86/6-31+G(d) & -0.2032 & -0.0463 & 0.1570 & 0.0785 & -12.7413 & 0.0992 & 0.2032 & 0.0463 & -0.1248 & 0.1248 \\
\hline
\end{tabular}




\begin{tabular}{|c|c|c|c|c|c|c|c|c|c|c|c|c|}
\hline & & B3LYP/6-31+G(d) & -0.2302 & -0.0164 & 0.2138 & 0.1069 & -9.3537 & 0.0711 & 0.2302 & 0.0164 & -0.1233 & 0.1233 \\
\hline & & B3LYP/6-311+G(d,p) & -0.2327 & -0.0194 & 0.2133 & 0.1067 & -9.3760 & 0.0745 & 0.2327 & 0.0194 & -0.1260 & 0.1260 \\
\hline & & pbepbe/6-31+G(d) & -0.2367 & -0.0953 & 0.1415 & 0.0707 & -14.1383 & 0.1948 & 0.2367 & 0.0953 & -0.1660 & 0.1660 \\
\hline & & $\mathrm{HF} / 6-31+\mathrm{G}(\mathrm{d})$ & -0.3723 & 0.0573 & 0.4296 & 0.2148 & -4.6552 & 0.0577 & 0.3723 & -0.0573 & -0.1575 & 0.1575 \\
\hline & Gas & bvp86/6-31+G(d) & -0.2407 & -0.0987 & 0.1420 & 0.0710 & -14.0845 & 0.2029 & 0.2407 & 0.0987 & -0.1697 & 0.1697 \\
\hline & & B3LYP/6-31+G(d) & -0.2782 & -0.0681 & 0.2101 & 0.1050 & -9.5202 & 0.1427 & 0.2782 & 0.0681 & -0.1732 & 0.1732 \\
\hline LQM & & B3LYP/6-311+G(d,p) & -0.2804 & -0.0719 & 0.2085 & 0.1042 & -9.5942 & 0.1488 & 0.2804 & 0.0719 & -0.1761 & 0.1761 \\
\hline 9010 & & pbepbe/6-31+G(d) & -0.2317 & -0.0899 & 0.1419 & 0.0709 & -14.0974 & 0.1822 & 0.2317 & 0.0899 & -0.1608 & 0.1608 \\
\hline & & $\mathrm{HF} / 6-31+\mathrm{G}(\mathrm{d})$ & -0.3627 & 0.0721 & 0.4348 & 0.2174 & -4.5996 & 0.0486 & 0.3627 & -0.0721 & -0.1453 & 0.1453 \\
\hline & PCM & bvp86/6-31+G(d) & -0.2350 & -0.0927 & 0.1423 & 0.0712 & -14.0548 & 0.1885 & 0.2350 & 0.0927 & -0.1638 & 0.1638 \\
\hline & & B3LYP/6-31+G(d) & -0.2710 & -0.0620 & 0.2090 & 0.1045 & -9.5689 & 0.1326 & 0.2710 & 0.0620 & -0.1665 & 0.1665 \\
\hline & & B3LYP/6-311+G(d,p) & -0.2760 & -0.0637 & 0.2123 & 0.1061 & -9.4224 & 0.1359 & 0.2760 & 0.0637 & -0.1699 & 0.1699 \\
\hline & & pbepbe/6-31+G(d) & -0.2230 & -0.0545 & 0.1685 & 0.0843 & -11.8666 & 0.1142 & 0.2230 & 0.0545 & -0.1387 & 0.1387 \\
\hline & & $\mathrm{HF} / 6-31+\mathrm{G}(\mathrm{d})$ & -0.2230 & -0.0545 & 0.1685 & 0.0843 & -11.8666 & 0.1142 & 0.2230 & 0.0545 & -0.1387 & 0.1387 \\
\hline & Gas & bvp86/6-31+G(d) & -0.2258 & -0.0570 & 0.1688 & 0.0844 & -11.8462 & 0.1184 & 0.2258 & 0.0570 & -0.1414 & 0.1414 \\
\hline & & B3LYP/6-31+G(d) & -0.2539 & -0.0277 & 0.2262 & 0.1131 & -8.8413 & 0.0877 & 0.2539 & 0.0277 & -0.1408 & 0.1408 \\
\hline LQM & & B3LYP/6-311+G(d,p) & -0.2558 & -0.0303 & 0.2256 & 0.1128 & -8.8660 & 0.0907 & 0.2558 & 0.0303 & -0.1431 & 0.1431 \\
\hline 9007 & & pbepbe/6-31+G(d) & -0.2262 & -0.0553 & 0.1709 & 0.0855 & -11.7000 & 0.1159 & 0.2262 & 0.0553 & -0.1408 & 0.1408 \\
\hline & & $\mathrm{HF} / 6-31+\mathrm{G}(\mathrm{d})$ & -0.2262 & -0.0553 & 0.1709 & 0.0855 & -11.7000 & 0.1159 & 0.2262 & 0.0553 & -0.1408 & 0.1408 \\
\hline & PCM & bvp86/6-31+G(d) & -0.2292 & -0.0579 & 0.1712 & 0.0856 & -11.6809 & 0.1203 & 0.2292 & 0.0579 & -0.1435 & 0.1435 \\
\hline & & B3LYP/6-31+G(d) & -0.2560 & -0.0281 & 0.2279 & 0.1139 & -8.7765 & 0.0885 & 0.2560 & 0.0281 & -0.1420 & 0.1420 \\
\hline & & B3LYP/6-311+G(d,p) & -0.2583 & -0.0311 & 0.2273 & 0.1136 & -8.8001 & 0.0921 & 0.2583 & 0.0311 & -0.1447 & 0.1447 \\
\hline & & pbepbe/6-31+G(d) & -0.2189 & -0.0576 & 0.1613 & 0.0806 & -12.4016 & 0.1185 & 0.2189 & 0.0576 & -0.1382 & 0.1382 \\
\hline & & $\mathrm{HF} / 6-31+\mathrm{G}(\mathrm{d})$ & -0.3374 & 0.0633 & 0.4007 & 0.2004 & -4.9910 & 0.0469 & 0.3374 & -0.0633 & -0.1370 & 0.1370 \\
\hline & $\begin{array}{l}\text { Gas } \\
\text { Phase }\end{array}$ & bvp86/6-31+G(d) & -0.2219 & -0.0603 & 0.1616 & 0.0808 & -12.3770 & 0.1232 & 0.2219 & 0.0603 & -0.1411 & 0.1411 \\
\hline & & B3LYP/6-31+G(d) & -0.2527 & -0.0306 & 0.2221 & 0.1110 & -9.0054 & 0.0903 & 0.2527 & 0.0306 & -0.1416 & 0.1416 \\
\hline LQM & & B3LYP/6-311+G(d,p) & -0.2547 & -0.0335 & 0.2213 & 0.1106 & -9.0383 & 0.0938 & 0.2547 & 0.0335 & -0.1441 & 0.1441 \\
\hline 9006 & & pbepbe/6-31+G(d) & -0.2188 & -0.0571 & 0.1617 & 0.0808 & -12.3724 & 0.1177 & 0.2188 & 0.0571 & -0.1379 & 0.1379 \\
\hline & & $\mathrm{HF} / 6-31+\mathrm{G}(\mathrm{d})$ & -0.3415 & 0.0713 & 0.4129 & 0.2064 & -4.8443 & 0.0442 & 0.3415 & -0.0713 & -0.1351 & 0.1351 \\
\hline & PCM & bvp86/6-31+G(d) & -0.2219 & -0.0598 & 0.1621 & 0.0811 & -12.3373 & 0.1224 & 0.2219 & 0.0598 & -0.1409 & 0.1409 \\
\hline & & B3LYP/6-31+G(d) & -0.2523 & -0.0302 & 0.2222 & 0.1111 & -9.0025 & 0.0898 & 0.2523 & 0.0302 & -0.1412 & 0.1412 \\
\hline & & B3LYP/6-311+G(d,p) & -0.2542 & -0.0332 & 0.2210 & 0.1105 & -9.0518 & 0.0934 & 0.2542 & 0.0332 & -0.1437 & 0.1437 \\
\hline $\begin{array}{c}\text { LQM } \\
9005\end{array}$ & Gas & pbepbe/6-31+G(d) & -0.2167 & -0.0501 & 0.1666 & 0.0833 & -12.0026 & 0.1068 & 0.2167 & 0.0501 & -0.1334 & 0.1334 \\
\hline & & $\mathrm{HF} / 6-31+\mathrm{G}(\mathrm{d})$ & -0.3334 & 0.0674 & 0.4007 & 0.2004 & -4.9910 & 0.0441 & 0.3334 & -0.0674 & -0.1330 & 0.1330 \\
\hline & & bvp86/6-31+G(d) & -0.2190 & -0.0526 & 0.1664 & 0.0832 & -12.0178 & 0.1108 & 0.2190 & 0.0526 & -0.1358 & 0.1358 \\
\hline & & B3LYP/6-31+G(d) & -0.2511 & -0.0221 & 0.2289 & 0.1145 & -8.7359 & 0.0815 & 0.2511 & 0.0221 & -0.1366 & 0.1366 \\
\hline & & B3LYP/6-311+G(d,p) & -0.2523 & -0.0250 & 0.2273 & 0.1136 & -8.8009 & 0.0846 & 0.2523 & 0.0250 & -0.1386 & 0.1386 \\
\hline & & pbepbe/6-31+G(d) & -0.2199 & -0.0501 & 0.1699 & 0.0849 & -11.7751 & 0.1073 & 0.2199 & 0.0501 & -0.1350 & 0.1350 \\
\hline & & $\mathrm{HF} / 6-31+\mathrm{G}(\mathrm{d})$ & -0.3364 & 0.0738 & 0.4102 & 0.2051 & -4.8761 & 0.0420 & 0.3364 & -0.0738 & -0.1313 & 0.1313 \\
\hline & PCM & bvp86/6-31+G(d) & -0.2229 & -0.0529 & 0.1700 & 0.0850 & -11.7668 & 0.1118 & 0.2229 & 0.0529 & -0.1379 & 0.1379 \\
\hline & & B3LYP/6-31+G(d) & -0.2525 & -0.0230 & 0.2294 & 0.1147 & -8.7169 & 0.0827 & 0.2525 & 0.0230 & -0.1377 & 0.1377 \\
\hline & & B3LYP/6-311+G(d,p) & -0.2548 & -0.0251 & 0.2297 & 0.1148 & -8.7078 & 0.0853 & 0.2548 & 0.0251 & -0.1400 & 0.1400 \\
\hline
\end{tabular}


Table 7. LD50 prediction results.

\begin{tabular}{cc}
\hline Compound & LD50 predicted $(\mathrm{mg} / \mathrm{kg})$ \\
\hline LQM9005 & 3500 \\
LQM9006 & 1480 \\
LQM9007 & 1540 \\
LQM9010 & 22.6 \\
LQM9011 & 1450 \\
\hline
\end{tabular}

Table 8. Statistic of the prediction model.

\begin{tabular}{ccc}
\hline Parameter & Value & Meaning \\
\hline $\mathrm{N}$ & 78 & Count of data points \\
$\mathrm{R}^{2}$ & 0.903 & Coefficient of determination \\
$\mathrm{R}^{2}$ adj & 0.898 & Adjusted coefficient of determination \\
$\mathrm{Q}^{2}$ & 0.889 & Coefficient of determination by "leave-one-out" validation \\
$\mathrm{SSR}$ & 0.639 & Sum of squared residuals \\
$\mathrm{S}$ & 0.0936 & Sample standard deviation of residuals \\
$\mathrm{F}$ & 171 & Fisher function \\
$\mathrm{Fa}$ & 2.97 & Fisher threshold for statistical significance (95\% confidence) \\
\hline
\end{tabular}

Table 9. Test set used to validate the QSAR model.

\begin{tabular}{|c|c|c|c|c|c|}
\hline Case & CAS & Name & $\begin{array}{c}\text { LD50 } \\
\text { Exp }\end{array}$ & $\begin{array}{l}\text { LD50 } \\
\text { Calc }\end{array}$ & REF \\
\hline 1. & $7492-44-6$ & (E)-3-(2-butylphenyl)acrylaldehyde & 4400 & 4370 & 19 \\
\hline 2. & $101-97-3$ & ethyl 2-phenylacetate & 3300 & 3440 & 20 \\
\hline 3. & $673-31-4$ & 3-phenylpropyl carbamate & 1110 & 1160 & 21 \\
\hline 4. & $\star 7-63-6$ & 2-oxobutyl 4-methylbenzenesulfonate & 1500 & 1590 & 22 \\
\hline 5. & $122-63-4$ & benzyl propionate & 3300 & 3470 & 23 \\
\hline 6. & $150-68-5$ & 3-(4-chlorophenyl)-1,1-dimethylurea & 1053 & 1360 & 25 \\
\hline 7. & $99-75-2$ & methyl-4-methylbenzoate & 3300 & 2960 & 26 \\
\hline 8. & $3060-89-7$ & 3-(4-bromophenyl)-1-methoxy-1-methylurea & 2000 & 1880 & 27 \\
\hline 10. & $5465-00-9$ & N-ethylbenzeneacetamide & 756 & 918 & 28 \\
\hline 11. & $103-93-5$ & propanoic acid, 2-methyl-,4-methylphenyl ester & 3970 & 3900 & 29 \\
\hline 12. & $52888-80-9$ & S-benzyl dipropylcarbamothioate & 1820 & 2130 & 30 \\
\hline 13. & $10402-52-5$ & 2-phenylpropyl acetate & 4290 & 3970 & 31 \\
\hline 14. & $538-32-9$ & benzylurea & 2700 & 2540 & 32 \\
\hline 15. & $104-64-3$ & 3-phenylpropyl formate & 4090 & 3510 & 33 \\
\hline 16. & $709-90-0$ & 1-phenylpropan-2-yl carbamate & 1100 & 1210 & 21 \\
\hline 17. & $1746-81-2$ & 3-(4-chlorophenyl)-1-methoxy-1-methylurea & 1800 & 1790 & 34 \\
\hline 18. & $1830-32-6$ & N,N-diethyl-2-((6-methylpyridazin-3-yl)thio)acetamide & 1550 & 1650 & 35 \\
\hline 19. & $122-72-5$ & 3-phenylpropyl acetate & 4700 & 3920 & 36 \\
\hline
\end{tabular}




\subsection{Spectroscopic Data}

LQM 9005: ethyl benzylcarbamate. Yellow solid pale, (yield 85\%), $\mathrm{mp} 42^{\circ} \mathrm{C}$ $44^{\circ} \mathrm{C}$, lit [18] $43^{\circ} \mathrm{C}-44^{\circ} \mathrm{C}$; IR (ATR, $\left.v, \mathrm{~cm}^{-1}\right) 3328.56(\mathrm{NH}), 2977.59(\mathrm{C}-\mathrm{H})$, $2922.50(\mathrm{C}-\mathrm{H}), 1695.83(\mathrm{C}=\mathrm{O}), 1525.72,(\mathrm{C}=\mathrm{C})$ 1245.36, 1136.90, 1034.21, 695.59. RMN 1H (300 MHZ, CDCl3, $\delta, \mathrm{ppm}): 7.327$ (5H, m H2, H3, H4), 5.20 $(1 \mathrm{H}$, brs, NH), $4.368(2 \mathrm{H}, \mathrm{d}, \mathrm{J}=6.0 \mathrm{~Hz}, \mathrm{H} 5), 4.173(2 \mathrm{H}, \mathrm{q}, \mathrm{J}=13.95 \mathrm{~Hz}, \mathrm{H} 7)$, $1.265(3 \mathrm{H}, \mathrm{t}, \mathrm{J}=13.95 \mathrm{~Hz}$ H8). RMN 13C (75.45 MHz, d-DMSO, $\delta, \mathrm{ppm})$ : 157.239 (C = O), 140.622 (C1), 128.92 (C3), 127.665 (C2), 127.408 (C4), 60.429 (C7), 44.387 (C5), 15.357 (C8). MS Ionization mode DART, Mass 180.10221 Mass calc for 180.10245, Formula for C10H14NO2 $(\mathrm{M}+1)$

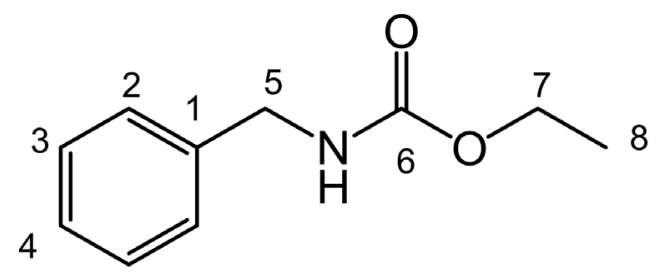

LQM 9006: ethyl 4-fluorobenzylcarbamate. White solid, yield 96.6\%, $\mathrm{mp} 60^{\circ} \mathrm{C}$ $61^{\circ} \mathrm{C}$. IR (ATR, $v \mathrm{~cm}^{-1}$ ) $3347.73(\mathrm{NH}), 2996.94,2943.11(\mathrm{C}-\mathrm{H}), 1683.60(\mathrm{C}=\mathrm{O})$, $1513.01(\mathrm{C}=\mathrm{C}), 1276.46,1218.04,1134.51,1039.37$ 828.89. RMN 1H (300 MHz; d-DMSO, $\delta, \mathrm{ppm}): 7.690(1 \mathrm{H}, \mathrm{t}, \mathrm{J}=6.0 \mathrm{~Hz} \mathrm{NH}), 7.307(2 \mathrm{H}, \mathrm{H} 2 \mathrm{~m}), 7.166(2 \mathrm{H}$, $\mathrm{H} 3 \mathrm{~m}), 4.167(2 \mathrm{H}, \mathrm{H} 5, \mathrm{~d}, \mathrm{~J}=6.0 \mathrm{~Hz}), 4.012(2 \mathrm{H}, \mathrm{q}, \mathrm{J}=14 \mathrm{~Hz}, \mathrm{H} 7), 1.183(3 \mathrm{H}, \mathrm{t}$, $\mathrm{J}=14 \mathrm{~Hz} \mathrm{H8})$. RMN 13C (75.45 MHz, d-DMSO, $\delta, \mathrm{ppm}): 157.269(\mathrm{C}=\mathrm{O})$, 144.251 (C4), 131.490 (C1), 128.481 (C2), 121.224 (C3), 60.716 (C7), 43.586 (C5), 15.191 (C8). MS Ionization mode DART, Mass 198.09223 Mass calc for 198.09269, formula for C10H13FNO2 $(\mathrm{M}+1)$

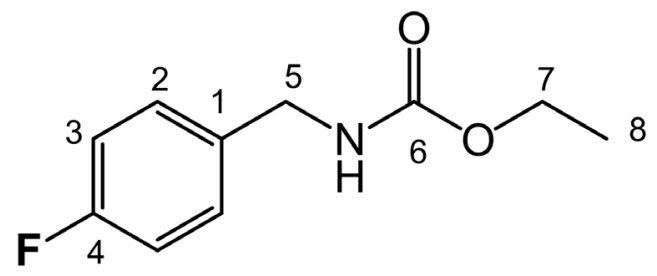

LQM 9007: ethyl 2-chlorobenzylcarbamate. Pale yellow solid, yield 86.3\%, mp $30^{\circ} \mathrm{C}-32^{\circ} \mathrm{C}$. IR (ATR, v, cm-1) 3297.26 (NH), $3062.11(\mathrm{CH}), 2977.29(\mathrm{CH})$, $2932.72(\mathrm{CH}), 1681.50(\mathrm{C}=\mathrm{O}), 1537.28(\mathrm{C}=\mathrm{C}), 1441.82,1284.07,1262.95$, $1154.16,1123.36,1033.47,926.60,749.19 . \mathrm{RMN} \mathrm{1H}(300 \mathrm{MHz}$; CDCl3; $\delta$, ppm): $7.383(4 \mathrm{H}, \mathrm{m}, \mathrm{H} 3, \mathrm{H} 4, \mathrm{H} 5, \mathrm{H} 6), 5.200(1 \mathrm{H}, \mathrm{NH}, \mathrm{brs}), 4.454(2 \mathrm{H}, \mathrm{d}, \mathrm{H} 7, \mathrm{~J}=6.3$ $\mathrm{Hz}), 4.158(2 \mathrm{H}, \mathrm{q}, \mathrm{H} 9 \mathrm{~J}=14.25 \mathrm{~Hz}), 1.259(3 \mathrm{H}, \mathrm{t}, \mathrm{H} 10, \mathrm{~J}=14.25 \mathrm{~Hz}) . \mathrm{RMN} 13 \mathrm{C}$ (75.45 MHz, d-DMSO, $\delta$, ppm): 157.193, (C = O), 137.372 (C1), 132.458 (C2), 129.767 (C3), 129.222 (C4), 129.056 (C5), 127.846 (C6), 60.625 (C9), 42.210 (C7), 15.327 (C10). MS Ionization mode DART, Mass 214.06412 Mass calc for 214.06318, formula for $\mathrm{C}_{10} \mathrm{H}_{13} \mathrm{ClNO}_{2}(\mathrm{M}+1)$ 


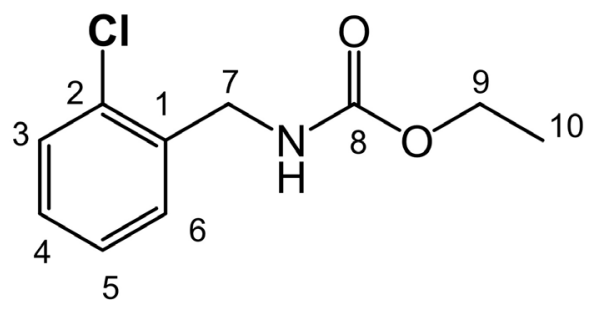

LQM 9010: ethyl 3,5-bis(trifluoromethyl) benzylcarbamate. White solid, yield 96.4\%, $\mathrm{mp} 80^{\circ} \mathrm{C}-81^{\circ} \mathrm{C}$. IR (ATR, $\left.v, \mathrm{~cm}^{-1}\right) 3315.21(\mathrm{NH}), 2989.39(\mathrm{CH}), 2939.34$ $(\mathrm{CH}), 1685.12,(\mathrm{C}=\mathrm{O}), 1535.58(\mathrm{C}=\mathrm{C}), 1382.57,1280.19,1252.70,1166.34$, $1121.12,1057.93,892.70,680.10$. RMN $1 \mathrm{H}(300 \mathrm{MHz}$; d-DMSO; $\delta$, ppm): 7.964 $(1 \mathrm{H}, \mathrm{s}, \mathrm{H} 4), 7.945(2 \mathrm{H}, \mathrm{s}, \mathrm{H} 2, \mathrm{H} 6) 7.848(1 \mathrm{H}, \mathrm{t}, \mathrm{NH}, \mathrm{J}=6.0 \mathrm{~Hz}), 4.382(2 \mathrm{H}, \mathrm{d}, \mathrm{H} 7$, $\mathrm{J}=6.0 \mathrm{~Hz}), 4.049(2 \mathrm{H}, \mathrm{q}, \mathrm{H} 9, \mathrm{~J}=14.25 \mathrm{~Hz}), 1.182(3 \mathrm{H}, \mathrm{t}, \mathrm{H} 10, \mathrm{~J}=14.25 \mathrm{~Hz})$. RMN 13C (75.45 MHz, d-DMSO, $\delta$, ppm): 163.438 (C9), 160.232 (C3), 157.193 $(\mathrm{C}=\mathrm{O}), 136.812(\mathrm{C} 1), 129.691(\mathrm{C} 2), 115.781$ (C4), 60.459(C7), 43.676 (C5), 15.312 (C8). MS Ionization mode DART, Mass 316.07808 Mass calc for 316.07722, formula for $\mathrm{C} 12 \mathrm{H} 12 \mathrm{~F} 6 \mathrm{NO} 2(\mathrm{M}+1)$

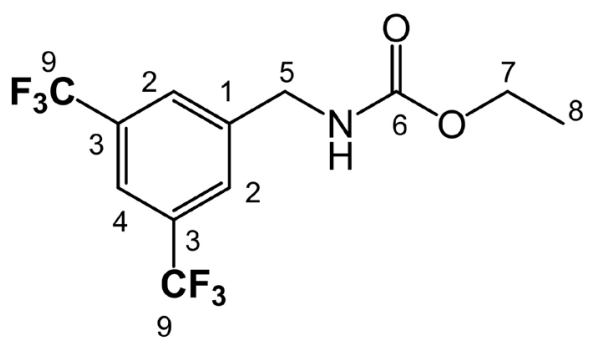

LQM 9011: ethyl 2-methoxybenzylcarbamate. Pale yellow foam, yield 92.6\%. IR (ATR, $\left.v, \mathrm{~cm}^{-1}\right)$ 3331.98(NH), $2978.97(\mathrm{CH}), 2932.45(\mathrm{CH}), 2837.64(\mathrm{CH})$, $1694.92(\mathrm{C}=\mathrm{O}), 1601,1514.90(\mathrm{C}=\mathrm{C}), 1491.80,1462.16,1237.42,1173.56$, $1120.20,1025.38,751.05 . \mathrm{RMN} 1 \mathrm{H}(300 \mathrm{MHz}$; d-DMSO; $\delta, \mathrm{ppm}): 7.483(1 \mathrm{H}, \mathrm{t}$, $\mathrm{NH}, \mathrm{J}=6.0 \mathrm{~Hz}), 7.251(2 \mathrm{H}, \mathrm{m}, \mathrm{H} 6, \mathrm{H} 5), 6.967(2 \mathrm{H}, \mathrm{m}, \mathrm{H} 3, \mathrm{H} 4), 4.171(2 \mathrm{H}, \mathrm{d}$, $\mathrm{H} 7, \mathrm{~J}=6.0 \mathrm{~Hz}), 4.017(2 \mathrm{H}, \mathrm{q}, \mathrm{H} 9, \mathrm{~J}=14.25 \mathrm{~Hz}), 3.783(3 \mathrm{H}, \mathrm{s}, \mathrm{H} 11), 1.194(3 \mathrm{H}, \mathrm{t}$, H10, J = 14.25 Hz). RMN 13C (75.45 MHz, d-DMSO, $\delta$, ppm): $157.208(\mathrm{C}=\mathrm{O})$, 157.087 (C2), 128.572 (C1), 127.892 (C6), 127.771 (C4), 120.740 (C5), 110.973 (C3), 60.383 (C9), 55.893 (C11), 40.713 (C7), 15.357 (C10). MS Ionization mode DART, Mass 210.11276 Mass calc for 210.11302, formula for C11H16NO3 (M + 1)

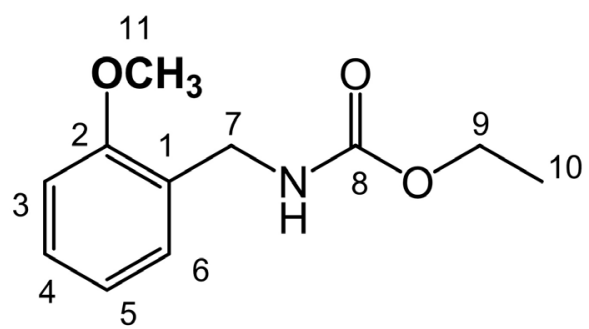




\section{Discussion}

\subsection{Vibrational Frequencies Analysis}

It is important to mention that although low levels of theory were used, the $\mathrm{HF} / 6-31+\mathrm{G}(\mathrm{d})$ represents a good commitment between the computational cost and the efficient performance of harmonic frequencies; since the electronic correlation methods do not offer significantly greater performance, the times of computational procedure are longer and they even present heavier errors [15].

The frequencies comparison was carried out with the most relevant bonds present in all carbamates, studied with the PM3 method, and theory levels HF/6-31+G(d), B3LYP/6-31+G(d), B3LYP/6-311+G(d,p), BVP86/6-31+G(d) and PBEPBE/6-31+G(d). presented in Tables 10-14.

For the next discussion, a 3\% is arbitrary defined as the maximum objective error, the HF method has the lowest error (80.00\% of computed frequencies under 3\% error), followed by B3LYP/6-31+G(d), B3LYP/6-311+G(d,p) and PBEPBE/6$31+\mathrm{G}(\mathrm{d})$ with $68.5 \%, 65.71 \%$ and $62.86 \%$ respectively. And the less accurate is PM3 with $48.57 \%$. Therefore, this last method, it is not considered as a useful one and there will not be further discussion about it and presented in Tables 10-14.

On the other hand, the $\mathrm{HF} / 6-31+\mathrm{G}(\mathrm{d})$ level produced very accurate results, having only troubles for the calculation of stretching $\mathrm{N}-\mathrm{H}$ bond, overestimating its value considerably. Though it happens the same with stretching $\mathrm{C}=\mathrm{O}$ bond, the generated error is much lower compared with stretching $\mathrm{N}-\mathrm{H}$ bond, leaving only the corresponding vibrations $v \mathrm{~N}-\mathrm{H}$. This method/basis set provided the minor error from the used sets, being this equal to $1.88 \% \pm 0.21 \%$. Also, HF has an RMS error of $12.81 \pm 0.21 \mathrm{~cm}^{-1}$, which is lightly superior to B3LYP/6-311++ $\mathrm{G}(\mathrm{d})$, which presents an RMS error of $12.10 \pm 0.28 \mathrm{~cm}^{-1}$. This last set also has trouble with $v \mathrm{~N}-\mathrm{H}$, overestimating this even more than $\mathrm{HF} / 6-31+\mathrm{G}(\mathrm{d})$. On the other hand, although it does not present complications with $v \mathrm{C}=\mathrm{O}$, it notably underestimates $v \mathrm{~N}-\mathrm{C}$, this triggers the average error to be higher. The $65.71 \%$ of compared vibrations are minor to a $3 \%$. This points out that although B3LYP/6$311+\mathrm{G}(\mathrm{d})$ has a RMS error lightly minor from $\mathrm{HF} / 6-31+\mathrm{G}(\mathrm{d})$, the ab initio method results on being better for the calculation of vibrational frequencies of studied compounds, by generating values with a lower percentage of error and require less time machine in an average of 06:27:29 $\mathrm{h}$ less than B3LYP/6-31+G(d).

According to the observations of the B3LYP/6-311+G (d,p) level, the obtained results point out it has the same complications than on B3LYP/6-31+G(d) underestimating even more the vibrations of same bonds. Although it is a bigger basis, most of the calculated frequencies has a lower accuracy than the B3LYP/6$31+G(d)$. This triggers an average error to a lower efficiency. Therefore, B3LYP/6$311+G(d, p)$ is not the best set for the calculation of vibrational frequencies of synthetized products. The obtained errors are widely similar (B3LYP) or minor (HF) to the ones reported previously [14] [15] [16]. 


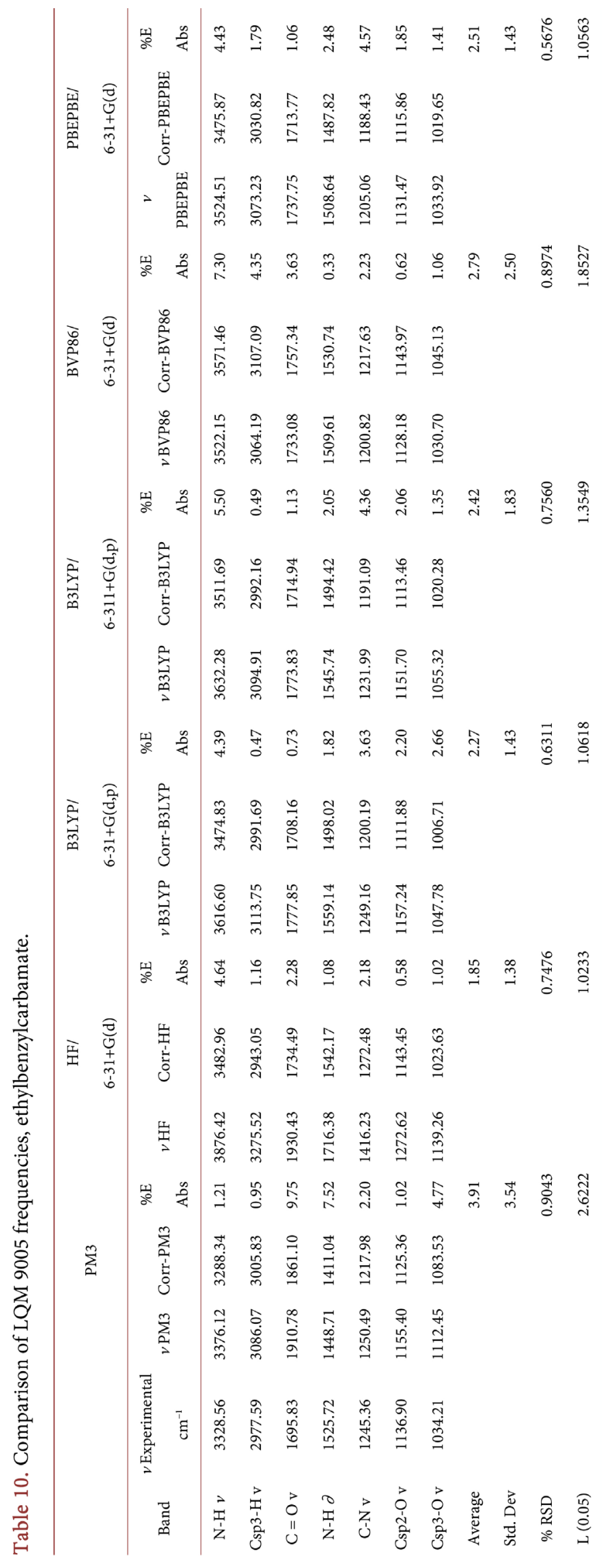


Table 11. Comparison of LQM 9006 Frequencies, ethyl 4-fluorobenzylcarbamate.

\begin{tabular}{|c|c|c|c|c|c|c|c|c|c|c|c|c|c|c|c|c|c|c|c|}
\hline & & & PM3 & & & $16-31+G($ & & B3LY & $\mathrm{P} / 6-31+\mathrm{C}$ & $\mathrm{G}(\mathrm{d})$ & B3LYP & $/ 6-311+C$ & $\mathrm{G}(\mathrm{d}, \mathrm{p})$ & BVP8 & $36 / 6-31+C$ & $G(d)$ & PBEPI & $\mathrm{BE} / 6-31+$ & $+\mathrm{G}(\mathrm{d})$ \\
\hline Band & $\begin{array}{l}v \text { Exp. } \\
\mathrm{cm}^{-1}\end{array}$ & $v$ & Corr & $\% \mathrm{E}_{\mathrm{Abs}}$ & $v$ & Corr & $\% \mathrm{E}_{\mathrm{Abs}}$ & $v$ & Corr & $\% \mathrm{E}_{\mathrm{Abs}}$ & $v$ & Corr & $\% \mathrm{E}_{\mathrm{Abs}}$ & $v$ & Corr & $\% \mathrm{E}_{\mathrm{Abs}}$ & $v$ & Corr & $\% \mathrm{E}_{\mathrm{Abs}}$ \\
\hline $\mathrm{N}-\mathrm{H} v$ & 3347.73 & 3360.36 & 3272.99 & 2.23 & 3865.63 & 3473.27 & 3.75 & 3615.03 & 3473.32 & 3.75 & 3629.51 & 3509.01 & 4.82 & 3515.35 & 3564.56 & 6.48 & 3523.85 & 3475.22 & 3.81 \\
\hline Csp3-H v & 2996.94 & 3184.85 & 3102.04 & 3.51 & 3275.99 & 2943.48 & 1.78 & 3134.78 & 3011.90 & 0.50 & 3114.24 & 3010.85 & 0.46 & 3030.15 & 3072.57 & 2.52 & 3073.84 & 3031.42 & 1.15 \\
\hline $\mathrm{C}=\mathrm{Ov}$ & 1683.60 & 1932.03 & 1881.80 & 11.77 & 1931.52 & 1735.47 & 3.08 & 1776.94 & 1707.28 & 1.41 & 1769.97 & 1711.21 & 1.64 & 1730.12 & 1754.34 & 4.20 & 1735.98 & 1712.02 & 1.69 \\
\hline N-H д & 1532.91 & 1457.27 & 1419.38 & 7.41 & 1716.23 & 1542.03 & 0.60 & 1559.46 & 1498.33 & 2.26 & 1545.66 & 1494.34 & 2.52 & 1505.87 & 1526.95 & 0.39 & 1506.93 & 1486.13 & 3.05 \\
\hline $\mathrm{C}-\mathrm{N} \mathrm{v}$ & 1262.17 & 1325.75 & 1291.28 & 2.31 & 1421.71 & 1277.41 & 1.21 & 1250.91 & 1201.87 & 4.78 & 1234.01 & 1193.04 & 5.48 & 1201.28 & 1218.10 & 3.49 & 1205.33 & 1188.70 & 5.82 \\
\hline Csp2-O v & 1143.32 & 1150.18 & 1120.28 & 2.02 & 1272.81 & 1143.62 & 0.03 & 1160.15 & 1114.67 & 2.51 & 1149.47 & 1111.31 & 2.80 & 1122.34 & 1138.05 & 0.46 & 1129.27 & 1113.69 & 2.59 \\
\hline Csp3-O v & 1031.74 & 1092.09 & 1063.70 & 3.10 & 1139.93 & 1024.23 & 0.73 & 1051.69 & 1010.46 & 2.06 & 1043.59 & 1008.94 & 2.21 & 1024.10 & 1038.44 & 0.65 & 1030.83 & 1016.60 & 1.47 \\
\hline Average & & & & 4.62 & & & 1.60 & & & 2.47 & & & 2.85 & & & 2.60 & & & 2.80 \\
\hline Std. Dev & & & & 3.66 & & & 1.37 & & & 1.43 & & & 1.75 & & & 2.30 & & & 1.63 \\
\hline$\%$ RSD & & & & 0.7920 & & & 0.8580 & & & 0.5782 & & & 0.6162 & & & 0.8840 & & & 0.5836 \\
\hline $\mathrm{L}(0.05)$ & & & & 2.7103 & & & 1.0144 & & & 1.0561 & & & 1.2992 & & & 1.7020 & & & 1.2091 \\
\hline
\end{tabular}

Table 12. Comparison of LQM 9007 Frequencies, ethyl 2-chlorobenzylcarbamate.

\begin{tabular}{|c|c|c|c|c|c|c|c|c|c|c|c|c|c|c|c|c|c|c|c|}
\hline \multirow[b]{2}{*}{ Band } & \multirow[b]{2}{*}{$\begin{array}{l}v \text { Exp. } \\
\mathrm{cm}^{-1}\end{array}$} & \multicolumn{3}{|c|}{ PM3 } & \multicolumn{3}{|c|}{$\mathrm{HF} / 6-31+\mathrm{G}(\mathrm{d})$} & \multicolumn{3}{|c|}{ B3LYP/6-31+G(d) } & \multicolumn{3}{|c|}{ B3LYP/6-311+G(d,p) } & \multicolumn{3}{|c|}{ BVP86/6-31+G(d) } & \multicolumn{3}{|c|}{ PBEPBE/6-31+G(d) } \\
\hline & & $v$ & Corr & $\% \mathrm{E}$ Abs & $v$ & Corr & $\begin{array}{l}\% \mathrm{E} \\
\mathrm{Abs}\end{array}$ & $v$ & Corr & $\begin{array}{l}\% \mathrm{E} \\
\mathrm{Abs}\end{array}$ & $v$ & Corr & $\% \mathrm{E}$ Abs & $v$ & Corr & $\%$ E Abs & $v$ & Corr & $\% \mathrm{E}$ Abs \\
\hline Csp3-H v & 2977.29 & 3086.12 & 3005.88 & 0.96 & 3276.13 & 2943.60 & 1.13 & 3114.17 & 2992.09 & 0.50 & 3095.47 & 2992.70 & 0.52 & 3064.71 & 3107.62 & 4.38 & 3073.50 & 3031.09 & 1.81 \\
\hline $\mathrm{C}=\mathrm{O} v$ & 1681.50 & 1908.79 & 1859.16 & 10.57 & 1931.44 & 1735.40 & 3.21 & 1777.25 & 1707.58 & 1.55 & 1770.00 & 1711.24 & 1.77 & 1740.72 & 1765.09 & 4.97 & 1747.10 & 1722.99 & 2.47 \\
\hline $\mathrm{C}-\mathrm{N} \mathrm{v}$ & 1262.95 & 1383.07 & 1347.11 & 6.66 & 1412.11 & 1268.78 & 0.46 & 1249.84 & 1200.85 & 4.92 & 1234.38 & 1193.40 & 5.51 & 1202.41 & 1219.24 & 3.46 & 1206.78 & 1190.13 & 5.77 \\
\hline Csp2-O v & 1123.36 & 1257.34 & 1224.65 & 9.02 & 1272.94 & 1143.74 & 1.81 & 1161.14 & 1115.62 & 0.69 & 1144.32 & 1106.33 & 1.52 & 1143.75 & 1159.76 & 3.24 & 1149.60 & 1133.74 & 0.92 \\
\hline Csp3-O v & 1024.16 & 1112.48 & 1083.56 & 5.80 & 1128.55 & 1014.00 & 0.99 & 1041.88 & 1001.04 & 2.26 & 1033.60 & 999.28 & 2.43 & 1034.07 & 1048.55 & 2.38 & 1039.53 & 1025.18 & 0.10 \\
\hline Average & & & & 7.11 & & & 1.70 & & & 2.63 & & & 3.07 & & & 3.87 & & & 2.81 \\
\hline Std. Dev & & & & 3.13 & & & 1.51 & & & 1.98 & & & 2.21 & & & 2.53 & & & 2.22 \\
\hline $\mathrm{L}(0.05)$ & & & & 2.3155 & & & 1.1204 & & & 1.4635 & & & 1.6344 & & & 1.8708 & & & 1.6435 \\
\hline
\end{tabular}

Table 13. Comparison of LQM 9010 Frequencies, ethyl 3,5-bis(trifluoromethyl)benzylcarbamate.

\begin{tabular}{|c|c|c|c|c|c|c|c|c|c|c|c|c|c|c|c|c|c|c|c|}
\hline \multirow[b]{2}{*}{ Band } & \multirow[b]{2}{*}{$\begin{array}{l}v \text { Exp. } \\
\mathrm{cm}^{-1}\end{array}$} & \multicolumn{3}{|c|}{ PM3 } & \multicolumn{3}{|c|}{$\mathrm{HF} / 6-31+\mathrm{G}(\mathrm{d})$} & \multicolumn{3}{|c|}{ B3LYP/6-31+G(d) } & \multicolumn{3}{|c|}{ B3LYP/6-311+G(d,p) } & \multicolumn{3}{|c|}{ BVP86/6-31+G(d) } & \multicolumn{3}{|c|}{ PBEPBE/6-31+G(d) } \\
\hline & & $v$ & Corr & $\% \mathrm{E}_{\mathrm{Abs}}$ & $v$ & Corr & $\% \mathrm{E}_{\text {Abs }}$ & $v$ & Corr & $\% \mathrm{E}_{\mathrm{Abs}}$ & $v$ & Corr & $\% \mathrm{E}_{\mathrm{Abs}}$ & $v$ & Corr & $\% \mathrm{E}_{\mathrm{Abs}}$ & $v$ & Corr & $\% \mathrm{E}_{\mathrm{Abs}}$ \\
\hline $\mathrm{N}-\mathrm{H} v$ & 3315.21 & 3373.95 & 3286.23 & 0.87 & 3869.75 & 3476.97 & 4.88 & 3622.18 & 3480.19 & 4.98 & 3635.50 & 3514.80 & 6.02 & 3522.62 & 3571.94 & 7.74 & 3529.94 & 3481.23 & 5.01 \\
\hline Csp3-H v & 2989.39 & 3085.92 & 3005.69 & 0.55 & 3279.49 & 2946.62 & 1.43 & 3117.06 & 2994.87 & 0.18 & 3073.19 & 2971.16 & 0.61 & 3066.91 & 3109.85 & 4.03 & 3040.41 & 2998.45 & 0.30 \\
\hline $\mathrm{C}=\mathrm{O} \mathrm{v}$ & 1685.12 & 1908.46 & 1858.84 & 10.31 & 1930.85 & 1734.87 & 2.95 & 1775.50 & 1705.90 & 1.23 & 1767.90 & 1709.21 & 1.43 & 1735.05 & 1759.34 & 4.40 & 1735.22 & 1711.27 & 1.55 \\
\hline $\mathrm{C}-\mathrm{N} v$ & 1252.70 & 1262.05 & 1229.24 & 1.87 & 1397.42 & 1255.58 & 0.23 & 1253.23 & 1204.10 & 3.88 & 1236.80 & 1195.74 & 4.55 & 1201.88 & 1218.71 & 2.71 & 1207.73 & 1191.06 & 4.92 \\
\hline Csp2-O v & 1119.35 & 1152.72 & 1122.75 & 0.30 & 1275.42 & 1145.96 & 2.38 & 1160.12 & 1114.64 & 0.42 & 1148.59 & 1110.46 & 0.79 & 1178.69 & 1195.19 & 6.78 & 1141.21 & 1125.46 & 0.55 \\
\hline Csp3-O v & 1057.93 & 1110.74 & 1081.86 & 2.26 & 1145.27 & 1029.03 & 2.73 & 1052.16 & 1010.92 & 4.44 & 1046.16 & 1011.43 & 4.40 & 1032.83 & 1047.29 & 1.01 & 1031.59 & 1017.35 & 3.84 \\
\hline Average & & & & 3.47 & & & 2.13 & & & 2.53 & & & 2.95 & & & 3.86 & & & 2.76 \\
\hline Std. Dev & & & & 4.04 & & & 1.63 & & & 1.96 & & & 2.10 & & & 2.76 & & & 1.98 \\
\hline$\%$ RSD & & & & 1.1633 & & & 0.7646 & & & 0.7770 & & & 0.7127 & & & 0.7139 & & & 0.7165 \\
\hline $\mathrm{L}(0.05)$ & & & & 2.9923 & & & 1.2088 & & & 1.4538 & & & 1.5573 & & & 2.0426 & & & 1.4634 \\
\hline
\end{tabular}


Table 14. Comparison of LQM 9011 frequencies, ethyl 2-methoxybenzylcarbamate.

\begin{tabular}{|c|c|c|c|c|c|c|c|c|c|c|c|c|c|c|c|c|c|c|c|}
\hline \multirow[b]{2}{*}{ Band } & \multirow[b]{2}{*}{$v$ Exp. $\mathrm{cm}^{-1}$} & \multicolumn{3}{|c|}{ PM3 } & \multicolumn{3}{|c|}{$\mathrm{HF} / 6-31+\mathrm{G}(\mathrm{d})$} & \multicolumn{3}{|c|}{ B3LYP/6-31+G(d) } & \multicolumn{3}{|c|}{ B3LYP/6-311+G(d,p) } & \multicolumn{3}{|c|}{ BVP86/6-31+G(d) } & \multicolumn{3}{|c|}{ PBEPBE/6-31+G(d) } \\
\hline & & $v$ & Corr & $\% \mathrm{E}_{\mathrm{Abs}}$ & $v$ & Corr & $\% \mathrm{E}_{\mathrm{Abs}}$ & $v$ & Corr & $\% \mathrm{E}_{\mathrm{Abs}}$ & $v$ & Corr & $\% \mathrm{E}_{\text {Abs }}$ & $v$ & Corr & $\% \mathrm{E}_{\mathrm{Abs}}$ & $v$ & Corr & $\% \mathrm{E}_{\text {Abs }}$ \\
\hline $\mathrm{N}-\mathrm{H} v$ & 3331.98 & 3378.29 & 3290.45 & 1.25 & 3887.32 & 3492.76 & 4.83 & 3624.83 & 3482.74 & 4.52 & 3638.07 & 3517.29 & 5.56 & 3525.47 & 3574.83 & 7.29 & 3534.96 & 3486.18 & 4.63 \\
\hline Csp3-H v & 2978.97 & 3086.09 & 3005.85 & 0.90 & 3272.91 & 2940.71 & 1.28 & 3087.28 & 2966.26 & 0.43 & 3066.79 & 2964.97 & 0.47 & 3054.71 & 3097.48 & 3.98 & 3065.55 & 3023.25 & 1.49 \\
\hline $\mathrm{C}=\mathrm{O} v$ & 1694.92 & 1906.52 & 1856.95 & 9.56 & 1931.62 & 1735.56 & 2.40 & 1778.73 & 1709.00 & 0.83 & 1771.20 & 1712.40 & 1.03 & 1739.51 & 1763.86 & 4.07 & 1746.06 & 1721.96 & 1.60 \\
\hline N-H $\partial$ & 1514.90 & 1451.67 & 1413.93 & 6.67 & 1708.21 & 1534.83 & 1.32 & 1551.50 & 1490.68 & 1.60 & 1538.79 & 1487.70 & 1.80 & 1514.29 & 1535.49 & 1.36 & 1516.73 & 1495.80 & 1.26 \\
\hline $\mathrm{C}-\mathrm{N} v$ & 1237.42 & 1267.03 & 1234.09 & 0.27 & 1401.85 & 1259.56 & 1.79 & 1287.32 & 1236.86 & 0.05 & 1228.33 & 1187.55 & 4.03 & 1201.19 & 1218.01 & 1.57 & 1205.52 & 1188.88 & 3.92 \\
\hline Csp2-O v & 1120.20 & 1156.72 & 1126.65 & 0.58 & 1271.54 & 1142.48 & 1.99 & 1155.94 & 1110.63 & 0.85 & 1146.27 & 1108.21 & 1.07 & 1147.12 & 1163.18 & 3.84 & 1146.56 & 1130.74 & 0.94 \\
\hline Csp3-O v & 1026.62 & 1112.72 & 1083.79 & 5.57 & 1129.48 & 1014.84 & 1.15 & 1044.43 & 1003.49 & 2.25 & 1036.94 & 1002.51 & 2.35 & 1034.34 & 1048.82 & 2.16 & 1039.67 & 1025.32 & 0.13 \\
\hline Average & & & & 3.54 & & & 2.11 & & & 1.50 & & & 2.33 & & & 3.47 & & & 1.99 \\
\hline$\%$ RSD & & & & 1.0429 & & & 0.6069 & & & 1.0098 & & & 0.7907 & & & 0.5902 & & & 0.8241 \\
\hline $\mathrm{L}(0.05)$ & & & & 2.7357 & & & 0.9474 & & & 1.1258 & & & 1.3644 & & & 1.5155 & & & 1.2175 \\
\hline
\end{tabular}

\subsection{Length and Angle Bond Analysis}

By comparing atomic bonds, a notable finding was that the lengths of all bonds $\mathrm{X}-\mathrm{H}$ (where $\mathrm{X}=\mathrm{C}, \mathrm{N}$ ) show error from $11 \%$ to $17 \%$, as all methods used, overestimate the lengths of such bonds between $0.08 \AA$ and $0.15 \AA$. Due to the preceding point, we decided to take all the other bonds left.

The HF method in general, predicts with a better accuracy the bonds C-C and $\mathrm{C}-\mathrm{N}$, while the DFT method using the functional B3LYP, calculates better the $\mathrm{C}-\mathrm{F}$ bond and two of the $\mathrm{C}-\mathrm{O}$ bonds. Even though the average error of B3LYP are minor than the $\mathrm{HF}$, the differences are not meaningful $[0.16 \%$ with $6-311+\mathrm{G}(\mathrm{d}, \mathrm{p})$ basis]. Considering that the HF did not overcome the $3 \%$ error and that the computational cost regards to $\mathrm{B} 3 \mathrm{LYP} / 6-311+\mathrm{G}(\mathrm{d}, \mathrm{p})$ is much lower, it is determined that the $\mathrm{HF} / 6-31+\mathrm{G}(\mathrm{d})$ is the best theory level to predict atomic bonds of LQM 9006 compound, having an average error of 1.14\%.

In regard of angles, the 5 sets provide really good results, with average error underneath $1 \%$. The HF method predicts most of the angles with better accuracy having the lowest average error: $0.53 \% \pm 0.13 \%$. Thus, the $\mathrm{HF} / 6-31+\mathrm{G}(\mathrm{d})$ basis is the most suitable method to predict atomic angles of LQM 9006.

The dihedral angles are not evaluated as they lack a conformational analysis of molecule, therefore a conformation of lower energy cannot be determined in the geometric minimizations. Nevertheless, since the imaginary frequencies were not observed, is assumed that the obtained geometries correspond to a local minimum and are useful for the comparison of vibrational frequencies, angles and bond lengths [12].

With the ChelpG data obtained the absolute percentage error $\left(\% \mathrm{E}_{\mathrm{Abs}}\right)$ was calculated for each theory level method, coming afterwards standard deviation (Std. Dev.), relative standard deviation (\%RSD) and Confidence Interval (CI $\alpha=$ 0.05) were calculated as well, Table 15. 
Table 15. Geometric structures comparison (Optimized vs Crystallographic) by ChelpG.

\begin{tabular}{cccccc}
\hline & pbepbe/6-31+G(d) & HF/6-31+G(d,) & bvp86/6-31+G(d) & B3LYP/6-31+G(d) & B3LYP/6-311+G(d,p) \\
\hline Average (\% Error) & 10.40 & 11.31 & 13.68 & 8.98 & 7.56 \\
Std. Dev. & 7.03 & 7.87 & 7.81 & 5.54 & 5.64 \\
\% RSD & 67.57 & 69.53 & 57.10 & 61.70 & 74.56 \\
CI (0.05) & 4.59 & 5.14 & 5.10 & 3.62 & 3.68 \\
\hline
\end{tabular}

By comparing the values of $\mathrm{E}_{\mathrm{HOMO}}$ and $\mathrm{E}_{\mathrm{LUmo}}$ given by the different theory levels, the following similarity can be noticed: the basis pbepbe/6-31+G(d) and bvp86/6-31+G(d) predict the energy values with a maximum difference of 0.005 $\mathrm{eV}$. The same happens between B3LYP/6-31+G(d) and B3LYP6-311+G(d,p). Thus, these similarities extend to the derived electronic descriptors calculated. The HF/6-31+G(d) method is the only basis that show a positive value in some of the $\mathrm{E}_{\mathrm{LUMO}}$ calculated therefore, the predicted data by this method is the one that varies the most.

The ionization potential (I) is a measure of the tendency of a molecule to donate electrons to an electron accepting specie. In the other hand the electron affinity (A) of a molecule is its tendency to accept electrons. The LQM molecules have an overall higher Ionization potential than Electron affinity and its tendency is:

$\mathrm{I}_{9010}>\mathrm{I}_{9006}>\mathrm{I}_{9005}>\mathrm{I}_{9007}>\mathrm{I}_{9011}$

The molecular orbital energy gap $(\Delta \mathrm{E})$ represents the stability of a compound. A larger $\Delta \mathrm{E}$ indicates that the compound is more stable, and it's highly related to the chemical hardness $(\eta)$ and chemical softness $(\sigma)$ : a hard molecule is said to have a large $\Delta \mathrm{E}$ value, and a soft molecule has small $\Delta \mathrm{E}$ value.

The tendency in stability is the next:

pbepbe and bvp 86: $9007<9005<9006<9011<9010$

Both B3LYP: $9005<9007<9006<9011<9010$

HF: $9010<9005=9006<9011<9007$

Chemical potential $(\mu)$ and electronegativity $(\chi)$ are intrinsically related $(\chi=$ $-\mu$ ) thus, one is often reported instead of the other. The overall tendency in ethyl carbamates regard Electronegativity is:

$9011<9005<9007,9006<9010$

By comparing the ChelpG charges between optimized and crystallographic geometry there seems to be an overall tendency of the atoms charge whether is positive or negative, except in the atoms that forms the aromatic ring structure. None of the methods estimate with precision the charge of those $\mathrm{C}_{\mathrm{sp} 2}$ atoms, thus to simplify the results the total charge sum of the aromatic ring was taken into account for the analysis (Table 16). The results showed that both DFT/B3LYP are the best methods to describe atomic charges despite their geometric optimization are not the best.

The Molecular Volume is a measure of the molecular contact surface (Table 17). The results display an overall tendency as expected: the more atoms the 
molecule has, higher the molecular volume. Mv: $9005<9006,9007<9011<$ 9010. Comparing the data between Gas phase and Polarizable continuum solvation model we observe that the molecular volume given by PCM is on average higher except with the B3LYP/6-311+G(d,p) method (Table 18).

Comparing the general results given by each of the basis functions, we could say that HF is the most accurate method to predict the geometry structure of a LQM9000 molecule but is unable to predict electronic data with high accuracy. Usually describing a chemical structure using theoretical calculations, take into consideration the quantum methods that offer a high level of theory, however the objective of carrying out theoretical calculations is to obtain optimized geometric structures so that the physicochemical parameters that are obtained are the most attached to a chemical reality (Table 19).

Although HF methods have ceased to be in force for its level of theory, to some chemical systems, carbamates in question, these methods showed better describe its geometry and validating them with critical parameters such as bond lengths, bond angles and frequencies of molecular vibration. For these chemical systems, carbamates, HF method showed a better description. Although the differences with the DFT methods are not large, we always take into account a method which describes the aforementioned parameters with better accuracy.

Table 16. ChelpG charged computed for the LQM series.

\begin{tabular}{|c|c|c|c|c|c|}
\hline & pbepbe/6-31+G(d) & $\mathrm{HF} / 6-31+\mathrm{G}(\mathrm{d})$ & bvp86/6-31+G(d) & B3LYP/6-31+G(d) & B3LYP/6-311+G(d,p) \\
\hline Compound & & & Carbamate moiet & & \\
\hline 9005 & -0.0086 & 0.0014 & -0.0055 & -0.0150 & -0.0048 \\
\hline 9006 & 0.0190 & 0.0312 & 0.0127 & 0.0288 & 0.0290 \\
\hline 9007 & 0.0488 & 0.0488 & 0.0479 & 0.0512 & 0.0492 \\
\hline 9010 & 0.0489 & 0.0456 & 0.0493 & 0.0385 & 0.0547 \\
\hline \multirow[t]{2}{*}{9011} & 0.0807 & 0.1066 & 0.0798 & 0.0871 & 0.0894 \\
\hline & & & Aromatic Ring & & \\
\hline 9005 & 0.0086 & -0.0014 & 0.0055 & 0.0150 & 0.0048 \\
\hline 9006 & 0.2200 & 0.2531 & 0.2246 & 0.2318 & 0.2351 \\
\hline 9007 & 0.0756 & 0.0756 & 0.0788 & 0.0873 & 0.0818 \\
\hline 9010 & 0.0700 & -0.0259 & 0.0683 & 0.0565 & -0.0191 \\
\hline \multirow[t]{2}{*}{9011} & 0.0555 & 0.0668 & 0.0527 & 0.0691 & 0.0567 \\
\hline & & & Substituent & & \\
\hline 9005 & 0.0000 & 0.0000 & 0.0000 & 0.0000 & 0.0000 \\
\hline 9006 & -0.2390 & -0.2843 & -0.2373 & -0.2606 & -0.2641 \\
\hline 9007 & -0.1245 & -0.1245 & -0.1267 & -0.1385 & -0.1311 \\
\hline 9010 & -0.1189 & -0.0198 & -0.1176 & -0.0950 & -0.0356 \\
\hline 9011 & -0.1362 & -0.1735 & -0.1326 & -0.1562 & -0.1460 \\
\hline
\end{tabular}


Table 17. Molecular volume, Diference Gas Phase - Solv $\left(\mathrm{cm}^{3} / \mathrm{mol}\right)$.

\begin{tabular}{cccccc}
\hline & pbepbe/6-31+G(d) & HF/6-31+G(d) & bvp86/6-31+G(d) & B3LYP/6-31+G(d) & B3LYP/6-311+G(d,p) \\
\hline 9005 & 19.315 & -12.520 & -21.259 & 9.148 & -6.167 \\
9006 & -6.902 & -12.638 & 2.440 & 41.379 & -33.777 \\
9007 & 50.078 & 43.231 & 6.672 & -30.293 & -9.698 \\
9010 & -45.489 & -1.022 & 23.198 & -15.590 & 4.156 \\
9011 & 12.332 & 3.970 & 49.214 & 9.086 & -19.000 \\
Average & 5.87 & 4.20 & 12.05 & 2.75 & -12.90 \\
\hline
\end{tabular}

Table 18. Molecular volume (Mv) calculated.

\begin{tabular}{|c|c|c|c|c|c|}
\hline LQM Code & pbepbe/6-31+G(d) & $\mathrm{HF} / 6-31+\mathrm{G}(\mathrm{d})$ & bvp86/6-31+G(d) & B3LYP/6-31+G(d) & B3LYP/6-311+G(d,p) \\
\hline \multicolumn{6}{|c|}{ Gas phase } \\
\hline 9005 & 144.047 & 137.993 & 155.593 & 134.587 & 157.160 \\
\hline 9006 & 153.694 & 151.318 & 148.036 & 112.994 & 194.664 \\
\hline 9007 & 144.403 & 135.234 & 151.620 & 158.235 & 146.636 \\
\hline 9010 & 222.230 & 163.388 & 193.418 & 191.244 & 196.406 \\
\hline 9011 & 148.428 & 150.866 & 155.694 & 149.997 & 174.956 \\
\hline \multicolumn{6}{|c|}{ Polarizable continuum solvation model } \\
\hline 9005 & 163.362 & 125.473 & 134.334 & 143.735 & 150.993 \\
\hline 9006 & 146.792 & 138.680 & 150.476 & 154.373 & 160.887 \\
\hline 9007 & 194.481 & 178.465 & 158.292 & 127.942 & 136.938 \\
\hline 9010 & 176.741 & 162.366 & 216.616 & 175.654 & 200.562 \\
\hline 9011 & 160.760 & 154.836 & 204.908 & 159.083 & 155.956 \\
\hline \multicolumn{6}{|c|}{ Diference Gas Phase - Solvation Model } \\
\hline 9005 & 19.315 & -12.520 & -21.259 & 9.148 & -6.167 \\
\hline 9006 & -6.902 & -12.638 & 2.440 & 41.379 & -33.777 \\
\hline 9007 & 50.078 & 43.231 & 6.672 & -30.293 & -9.698 \\
\hline 9010 & -45.489 & -1.022 & 23.198 & -15.590 & 4.156 \\
\hline 9011 & 12.332 & 3.970 & 49.214 & 9.086 & -19.000 \\
\hline Average & 5.87 & 4.20 & 12.05 & 2.75 & -12.90 \\
\hline
\end{tabular}

Table 19. Amino-dehalogenation of alkyl chloroformates differences.

\begin{tabular}{ccccc}
\hline Reference & Temperature $\left({ }^{\circ} \mathrm{C}\right)$ & catalyst & solvent & Yield (\%) \\
\hline 36 & 0 & $\mathrm{NaOH}$ & $\mathrm{H}_{2} \mathrm{O} / \mathrm{Et}_{2} \mathrm{O}$ & 75 \\
37 & 25 & Lewis acid Y-Zr & $\mathrm{MeCN}$ & 90 \\
38 & 25 & $\mathrm{Zn}$ & Benzene & 97 \\
40 & 25 & $\mathrm{Et}_{3} \mathrm{~N}$ & $\mathrm{THF}$ & 43 \\
41 & $10-15$ & $\mathrm{NaOH}_{2} \mathrm{O}$ & 97 \\
42 & Reflux/IR & $\mathrm{NaHCO}_{3}$ & Acetone & 67.84 \\
\hline
\end{tabular}




\subsection{LD50 Prediction Calculations}

LD50 prediction showed that LQM9005, LQM9006, LQM9007 and LQM9011 compounds are relatively safe. This is because of the high levels needed to induce lethality. However, the LQM9010 showed to be more unsafe than the other compounds. These results are supported with the statistical parameters needed to validate a QSAR model, such as an $\mathrm{R}^{2}$ higher than 0.7 and the difference between $\mathrm{R}^{2}$ and $\mathrm{Q}^{2}$ shouldn't be higher than 0.3 . Also, the number of compounds used to generate the model are 78 points. And the model showed to be robust enough according with the validation parameter $\mathrm{Q}^{2}$. The test set corroborates how robust is the model. In this case there is an $\mathrm{R}^{2}=0.9647$, showing how does the predictive level of the equation correlates with a high level of confidence.

\subsection{Synthesis}

The Aliphatic carbamates synthesis has been made during the last years [37]-[63]. In these examples different changes are made, changing the nature of the base, solvent or temperature; giving a different efficiency and compares the different variations upon the analyzed reaction, as it is shown in some examples are described in Table 19.

The methodology described in this work had not previously been used and has advantages over those previously reported, specifically focused on the synthesis of ethylbenzyl carbamate and very similar compounds, which consisted in using a stronger basis $\left(\mathrm{K}_{2} \mathrm{CO}_{3}\right)$, with no need to increase temperature, making the process under ambient conditions and the yields obtained are excellent from $86 \%$ to $96 \%$. The reason why a stronger basis than $\mathrm{NaHCO}_{3}$ is required, is because of the difference in constants of acidity of aromatic and aliphatic amines: while the first represents a pKa of approximately $4.5\left(\mathrm{pKaPhNH}_{3}+/ \mathrm{PhNH}_{2}=\right.$ 4.63), second possess a $\mathrm{pKa}$ around $10.5\left(\mathrm{pKaMeNH}_{3}+/ \mathrm{MeNH}_{2}=10.6\right)$. In comparison, used bases possess the next values, $\mathrm{pKaH}_{2} \mathrm{CO}_{3} / \mathrm{HCO}_{3}^{-}=6.35$, against $\mathrm{pKa} \mathrm{HCO}_{3}^{-} / \mathrm{CO}_{3}^{2-}=10.2 .{ }^{39}$ Even though the values are referred to the water environment, it is useful to compare the relative basicity of each kind of compounds, being, in case of the amines shown with a deeply great difference, as methylamine is 105.97 times more basic than aniline. It can also be observed because of the $\mathrm{HCO}^{3-}$ ion is an efficient base by using it in the aromatic carbamate synthesis, being more basic than the aromatic amines. Besides it is being considerably less basic than aliphatic amine, is not useful to function as a base (on the contrary, it acts as an acid) with the mentioned substrates, requiring the IR radiation appliance to be used. On the contrary, $\mathrm{CO}_{3}{ }^{2-}$ ion has a relative basicity very like aliphatic amines, therefore they can be neutralized, nevertheless, to benefit its efficiency it is necessary to use a higher quantity according to the stoichiometric balance (Equation (2)).

$$
\mathrm{RNH}_{3} \mathrm{Cl}+\mathrm{K}_{2} \mathrm{CO}_{3} \rightleftarrows \mathrm{RNH}_{2}+\mathrm{KHCO}_{3}+\mathrm{KCl}
$$

The previous equation corresponds to the neutralization of an ammoniac salt 
by the potassium carbonate. Even though the basicity that the amine and the carbonate present is very similar, according to Le Châtelier principle, it benefited the reaction of neutralization in our favor, by increasing the stoichiometric quantity of potassium carbonate. Another fact that benefited the reaction, was the gradual consumption of amine as the synthesis of carbamate passed. Five compounds were chosen for its synthesis, modifying the structure with the increase of one carbon atom such as benzylamine and its derivatives, with electron donors and electron acceptors, which could allow the effect evaluation of this substituent upon its properties, considering that the synthetized structures are hydrophobic and none of them represent a meaningful steric effect.

\section{Conclusion}

The vibrational frequencies of synthesized compounds were calculated, using the PM3 semi empirical method, the method ab initio HF with $6-31+\mathrm{G}(\mathrm{d})$ function, the DFT method, using the hybrid functional B3LYP with $6-31+\mathrm{G}(\mathrm{d})$ and $6-311+G(d, p)$ functions, the basis pbepbe/6-31+G(d) and bvp86/6-31+G(d). The calculated frequencies were scaled with the appropriate factors and were compared with the ones observed experimentally. The level of theory HF/6-31+G(d) was calculated with the intention of providing closer values to the experimental ones $\left(\% \mathrm{E}_{\mathrm{Abs}}=1.88 \% \pm 0.26 \%, \mathrm{RM}_{\mathrm{Sov}}=32.88 \pm 0.23 \mathrm{~cm}^{-1}\right)$ and requiring less time for the computational procedure, which is the best prediction of vibrational frequencies of the synthesized compounds. Additionally, lengths and theoretical and experimental angles of the bonds were compared from the LQM 9006 model [(4-fluorobenzyl) ethyl carbamate]; once again, the $a b$ initio method was the most effective for the calculation of such properties, even it did not provide the results of minor error (for bond lengths), the values caused by the other methods were not very different, so therefore by considering the computational time HF is the most suitable method. Synthesis and relative safety of new 5 different ethyl benzyl carbamates was carried out, and it was clear that the synthetic methodology applied was better; in addition the reaction is performed at room temperature with excellent reaction yields. Also the computed endpoint (LD50) shows us that the proposed compounds are safe enough due to the high doses needed to trigger an acute toxicity response.

\section{Acknowledgements}

We would like to thank, D. Jimenez, M. Hernandez from FESC-UNAM and for their skillful technical assistance, CONACyT, Posgrado en Ciencias Biomedicas UNAM. Proyect: PAPIIT IT200817, PAPIIT No 202015, Catedra PIAPI1607: Diseno de moleculas bioativas.

\section{Conflicts of Interest}

The authors declare no conflicts of interest regarding the publication of this paper. 


\section{References}

[1] The Center for the Food Security \& Public Health (2007) Rhipicephalus microplus. Iowa State University.

http://www.cfsph.iastate.edu/Factsheets/pdfs/boophilus_microplus.pdf

[2] Servicio Nacional de Sanidad, Inocuidad y Calidad Agroalimentaria (2013) Garrapata Boophilus spp.

[3] http://www.senasica.gob.mx/?id=4373

[4] Rodriguez, V. (2011) Epidemiologia y Control de Rhipicephalus (Boophilus microplus) en Mexico. Epidemiologia de Enfermedades Parasitarias en Animales Domesticos. Electronic pressMexico.

[5] SAGARPA (2015) Secretaría de agricultura, ganadería, desarrollo rural pesca y alimentación.

[6] https://www.gob.mx/sagarpa

[7] Perez, L.C., Rodriguez, V.R.I., Ramirez, C.G.T. and Miller, R.J. (2010) First Report of the Cattle Tick Rhipicephalus microplus Resistant to Ivermectin in Mexico. Veterinary Parasitology, 168, 165-169. https://doi.org/10.1016/j.vetpar.2009.10.021

[8] George, J.E., Pound, J.M. and Davey, R.B. (2004) Chemical Control of Ticks on Cattle and the Resistance of these Parasites to Acaricides. Parasitology, 129, S353-S366. https://doi.org/10.1017/S0031182003004682

[9] Iturbe, R.S.L. (2014) Evaluacion de la Eficacia in vivo de Dos Carbamatos de Nueva Sintesis sobre Rhipicephalus microplus. MSc dissertation, UNAM. FES Cuautitlán, Mexico.

[10] Helguera, A.M., Alfonso, P.G., Alexandra, G., Joana, R., Fernando, C., Dolores, V., Cordeiro, M. and Fernanda, B. (2013) Combining QSAR Classification Models for Predictive Modeling of Human Monoamine Oxidase Inhibitors. European Journal of Medicinal Chemistry, 59, 75. https://doi.org/10.1016/j.ejmech.2012.10.035

[11] Prado, O.M.G., Alba, H.F., Angeles E., Velazquez, S.A.M., Ramirez, N.P., Diaz, T.R. and Munoz, G.M.A. (2014) The Action of Two Ethyl Carbamates on Acetylcholinesterase and Reproductive Organs of Rhipicephalus microplus. Veterinary Parasitology, 199, 215. https://doi.org/10.1016/j.vetpar.2013.10.028

[12] Gaussian 16, Revision B.01, Frisch, M.J., Trucks, G.W., Schlegel, H.B., Scuseria, G.E., Robb, M.A., Cheeseman, J.R.; Scalmani, G.; Barone, V.; Petersson, G.A.; Nakatsuji, H.; Li, X.; Caricato, M.; Marenich, A.V.; Bloino, J., Janesko, B.G., Gomperts, R., Mennucci, B., Hratchian, H.P., Ortiz, J.V., Izmaylov, A.F., Sonnenberg, J.L., Williams-Young, D., Ding, F., Lipparini, F., Egidi, F., Goings, J., Peng, B., Petrone, A., Henderson, T., Ranasinghe, D., Zakrzewski, V.G., Gao, J., Rega, N., Zheng, G., Liang, W., Hada, M., Ehara, M., Toyota, K., Fukuda, R., Hasegawa, J., Ishida, M., Nakajima, T., Honda, Y., Kitao, O., Nakai, H., Vreven, T., Throssell, K., Montgomery Jr., J.A., Peralta, J.E., Ogliaro, F., Bearpark, M.J., Heyd, J.J., Brothers, E.N., Kudin, K.N., Staroverov, V.N., Keith, T.A., Kobayashi, R., Normand, J., Raghavachari, K., Rendell, A.P., Burant, J.C., Iyengar, S.S., Tomasi, J., Cossi, M., Millam, J.M., Klene, M., Adamo, C., Cammi, R., Ochterski, J.W., Martin, R.L., Morokuma, K., Farkas, O., Foresman, J.B., Fox, D.J. Gaussian, Inc., Wallingford CT (2016) GaussView 5.0. Wallingford, E.U.A.

[13] Lewars, E.G. (2011) Computational Chemistry: Introduction to the Theory and Applications of Molecular and Quantum Mechanics. Springer. Netherlands.

[14] Cuevas, G. and Cortes, F. (2003) Introducción a la Química Computacional (1a ed.). Fondo de Cultura Economica, Mexico. 
[15] Andersson, M.P. and Uvdal, P. (2005) New Scale Factors for Harmonic Vibrational Frequencies Using the B3LYP Density Functional Method with the Triple-Z Basis Set 6-311+(d,p). Journal of Physical Chemistry A, 109, 2937. https://doi.org/10.1021/jp045733a

[16] Scott, A.P. and Radom, L. (1996) Harmonic Vibrational Frequencies: An Evaluation of Hartree-Fock, Moller-Plesset, Quadratic Configuration Interaction, Density Functional Theory, and Semiempirical Scale Factors. Journal of Physical Chemistry, 100, 16502-16513. https://doi.org/10.1021/jp960976r

[17] Merrick, J.P., Moran, D. and Radom, L. (2007) An Evaluation of Harmonic Vibrational Frequency Scale Factors. Journal of Physical Chemistry A, 111, 11683-11700. https://doi.org/10.1021/jp073974n

[18] Sheldrick, G.M. (2008) A Short History of SHELX. Acta Crystallographica A, 64, 112-122. https://doi.org/10.1107/S0108767307043930

[19] (2012) OECD QSAR Application Toolbox, Ver. 3.4. https://www.qsartoolbox.org/home

[20] Casadei, M.A., Moracci, F.M., Zappia, G. and Rossi, L. (1997) Electrogenerated Superoxide-Activated Carbon Dioxide. A New Mild and Safe Approach to Organic Carbamates. Journal of Organic Chemistry, 62, 6754-6759. https://doi.org/10.1021/jo970308h

[21] (1980) Monograph, Food and Cosmetics Toxicology. 657.

[22] (1975) Monograph, Food and Cosmetics Toxicology. 99.

[23] Stille, G., Wagner-Jauregg, T. and Haering, M. (1963) Central Muscle Relaxants. Drug Research. 856.

[24] Setsuro, F., Toshihiro, H., Kazuo, O., Yoshiyuki, M. and Sadao, H. (1981). https://chem.nlm.nih.gov/chemidplus/rn/101-97-3

[25] (1975) Monograph, Food and Cosmetics Toxicology. 723.

[26] Gaines, T. and Linder, L. (1986) Acute Toxicity of Pesticides in Adult and Weanling Rats. Fundamental and Applied Toxicology, 7, 299-308. https://doi.org/10.1016/0272-0590(86)90160-0

[27] (1979) Monograph, Food and Cosmetics Toxicology. 873.

[28] (1970) World Review of Pest Control. 119. https://chem.nlm.nih.gov/chemidplus/rn/3060-89-7

[29] (1988) Monograph, Food and Chemical Toxicology. 287.

[30] (1997) Chinese Pharmaceutical Journal, 135. https://chem.nlm.nih.gov/chemidplus/rn/5465-00-9

[31] (1975) Monograph, Food and Cosmetics Toxicology. 773.

[32] (1991) Pesticide Manual. 729. https://chem.nlm.nih.gov/chemidplus/rn/52888-80-9

[33] (1976) Monograph, Food and Cosmetics Toxicology. 797.

[34] (1979) HYSAAV. 68. https://chem.nlm.nih.gov/chemidplus/rn/538-32-9

[35] (1976) Monograph, Food and Cosmetics Toxicology. 835.

[36] (1970) World Review of Pest Control. 119. https://chem.nlm.nih.gov/chemidplus/rn/1746-81-2

[37] Stormann, H. (1964) Choleretic and Other Pharmacological Properties of 2-(3chloro-6-pyridazinylthio)-acetic Acid Diethylamide, Drug Research. 266.

[38] (1974) Food and Cosmetics Toxicology. Monographs. Fragrance Raw Materials 
Monographs: Phenylpropyl Acetate. 965.

[39] March, J. and Smith, M.B. (2007) March's Advanced Organic Chemistry. Reactions, Mechanisms, and Structure. Wiley, Hoboken.

[40] Fukuoka, S., Chono, M. and Kohno, M. (1984) A Novel Catalytic Synthesis of Carbamates by Oxydative Alkoxycarbonylation of Amines in the Presence of Palladium and Iodide. Journal of the Chemical Society, Chemical Communications, 399-400. https://doi.org/10.1039/c39840000399

[41] Chatuverdi, D., Mishra, N. and Mishra, V. (2007) An Efficient, One-Pot Synthesis of Carbamates from the Corresponding Alcohols Using Mitsunobu's Reagent. Mo natshetfte für Chemie, 138, 57-60. https://doi.org/10.1007/s00706-006-0557-2

[42] Inesi, A., Feroci, M. and Rossi, L. (2000) The Reaction of Amines with an Electrogenerated Base. Improved Synthesis of Arylcarbamic Esters. Tetrahedron Letters, 41, 963-966. https://doi.org/10.1016/S0040-4039(99)02180-2

[43] Perez, E., da Silv, M.O., Costa, V., Rodrigues-Fihlo, U. and Franco, D. (2002) Efficient and Clean Synthesis of N-Alkyl Carbamates by Transcarboxylation and O-Alkylation Coupled Reactions Using a DBU- $\mathrm{CO}_{2}$ Zwitterionic Carbamic Complex in Aprotic Polar Media. Tetrahedron Letters, 43, 4091-4093. https://doi.org/10.1016/S0040-4039(02)00697-4

[44] Chaturvedi, D. and Ray, S. (2006) Versatile Use of Carbon Dioxide. Monatshefte für Chemie, 137, 127-145. https://doi.org/10.1007/s00706-005-0423-7

[45] Cenini, S. and Ragaini, F. (2013) Catalytic Reductive Carbonylation of Organic Nitro Compounds (Vol. 20 of Catalysis by Metal Complexes). Springer Science \& Business Media, Berlin.

[46] Fu, X., Zhang, Z., Li, C., Wang, L., Ji, H., Yang, Y. and Gao, G. (2009) N-Heterocyclic Carbomethoxylation Catalized by Ionic Liquids in the Presence of Dimethyl Carbonate. Catalysis Communications, 10, 665-668. https://doi.org/10.1016/j.catcom.2008.11.012

[47] Zhou, H., Shi, F., Tian, X., Zhang, Q. and Deng, Y. (2007) Synthesis of Carbamates from Aliphatic Amines and Dimethyl Carbonate Catalyzed by Acid Functional Ionic Liquids. Journal of Molecular Catalysis A: Chemical, 271, 89-92. https://doi.org/10.1016/j.molcata.2007.02.017

[48] Yang, X., Zhang, Y. and Ma, D. (2012) Synthesis of Aryl Carbamates via Copper-Catalyzed Coupling ofAryl Halides with Potassium Cyanate. Advanced Synthesis \& Catalysis, 354, 2443-2446. https://doi.org/10.1002/adsc.201200296

[49] Kainmehr, E. and Baghersad, M.H. (2011) Copper-Catalyzed Coupling of Arylboronic Acids with Potassium Cyanate: A New Approach to the Synthesis of Aryl Carbamates. Advanced Synthesis \& Catalysis, 353, 2599-2603. https://doi.org/10.1002/adsc.201100153

[50] Sandler, S.R. and Karo, W. (2013) Organic Functional Group Preparations. Elsevier, Berlin.

[51] Santi, R., Romano, A., Panella, F., Mestroni, G., Sessanti, A. and Santi, O. (1999) Reductive Carbonylation of 2,4-Dinitrotoluene to 2,4-Toluendiurethane with Palla$\operatorname{dium}(1,10$-phenanthroline)2(hexafluorophosphate)2, as Catalyst, and 1,10-Phenanthrolinium Hexafluorophosphate, as Cocatalyst. Journal of Molecular Catalysis A: Chemical, 144, 41-45. https://doi.org/10.1016/S1381-1169(98)00379-3

[52] Krogul, A., Skupińska, J. and Litwinienko, G. (2011) Catalytic Activity of $\mathrm{PdCl}_{2}$ Complexes with Pyridines in Nitrobenzene Carbonylation. Journal of Molecular Catalysis A: Chemical, 337, 9-16. https://doi.org/10.1016/j.molcata.2011.01.007 
[53] Chaturvedi, D. (2012) Perspectives on the Synthesis of Organic Carbamates. Tetrahedron, 68, 15-45. https://doi.org/10.1016/j.tet.2011.10.001

[54] D’Addona, D. and Bochet, C.G. (2001) Preparation of Carbamates from Amines and Alcohols under Mild Conditions. Tetrahedron Letters, 42, 5227-5229. https://doi.org/10.1016/S0040-4039(01)00991-1

[55] Chassaing, C., Berger, M., Heckeroth, A., Ilg, T., Jager, M., Kern, C. and Uphoff, M. (2008) Highly Water-Soluble Prodrugs of Anthelmintic Benzimidazole Carbamates: Synthesis, Pharmacodynamics, and Pharmacokinetics. Journal of Medicinal Chemistry, 51, 1111-1114. https://doi.org/10.1021/jm701456r

[56] Cooper, C.S., Peyton, A.L. and Weinkam, R.J. (1983) Formation Nitrosamines by Alkylation of Diazotates. Journal of Organic Chemistry, 48, 4116-4119. https://doi.org/10.1021/jo00170a052

[57] Pandey, R.K., Dagade, S.P., Dongare, M.K. and Kumar, P. (2003) Synthesis of Carbamates Using Yttria-Zirconia Based Lewis Acid Catalyst. Synthetic Communications, 33, 4019-4027. https://doi.org/10.1081/SCC-120026337

[58] Yadav, J.S., Reddy, G.S., Reddy, M.M. and Meshram, H.M. (1998) Zinc Promoted Simple and Convenient Synthesis of Carbamates: An Easy Access for Amino Group Protection. Tetrahedron Letters, 39, 3259-3262. https://doi.org/10.1016/S0040-4039(98)00464-X

[59] Solomons, G., Fryhle, C. and Snyder, S. (2014) Organic Chemistry. John Wiley \& Sons, Hoboken.

[60] Bach, A., Stuhr-Hansen, N., Thorsen, T.S., Bork, N., Moreira, I.S., Frydenvang, K. and Weinstein, H. (2010) Structure-Activity Relationships of a Small-Molecule Inhibitor of the PDZ Domain of PICK1. Organic \& Biomolecular Chemistry, 8, 4281-4288. https://doi.org/10.1039/c0ob00025f

[61] Wang, X., Lou, Q., Guo, Y., Xu, Y., Zhang, Z. and Liu, J. (2006) The Design and Synthesis of 9-Phenylcyclohepta[d]pyrimidine-2,4-dione Derivatives as Potent Non-Nucleoside Inhibitors of HIV Reverse Transcriptase. Organic \& Biomolecular Chemistry, 4, 3252-3258. https://doi.org/10.1039/b607972p

[62] Muñoz, C.C.E. (2012) Sintesis de Derivados del Acido Carbamico de la Serie LQM-900, los cuales han Mostrado Actividad Ixodicida. Undergraduate Dissertation, UNAM, FES Cuautitlan, Mexico.

[63] Avila, S.B.L. (2014) Sintesis de 5 Nuevos Carbamatos con Actividad Ixodicida en Garrapatas del Genero Boophilus. Tesis de Licenciatura, Undergraduate Dissertation, UNAM, FES Cuautitlan, Mexico. 